\title{
Small-amplitude grain boundaries of arbitrary angle in the Swift-Hohenberg equation
}

\author{
Arnd Scheel and Qiliang Wu \\ University of Minnesota, School of Mathematics \\ 206 Church St. S.E., Minneapolis, MN 55455, USA
}

September 7, 2012

\begin{abstract}
We study grain boundaries in the Swift-Hohenberg equation. Grain boundaries arise as stationary interfaces between roll solutions of different orientations. Our analysis shows that such stationary interfaces exist near onset of instability for arbitrary angles between the roll solutions. This extends prior work in [6] where the analysis was restricted to large angles, that is, weak bending near the grain boundary. The main new difficulty stems from possible interactions of the primary modes with other resonant modes. We generalize the normal form analysis in [6] and develop a singular perturbation approach to treat resonances.
\end{abstract}

Acknowledgments This work was partially supported by the National Science Foundation through grant NSF-DMS-0806614.

Running head: Grain boundaries in the Swift-Hohenberg equation

Corresponding author: Arnd Scheel

Keywords: Swift-Hohenberg equation, grain boundary, spatial dynamics, normal forms, singular perturbation 


\section{Introduction}

Rayleigh-Bénard convection has been one of the prime motivating examples of research in pattern formation. Much insight into the dynamics has been gained by formal or rigorous approximation of the dynamics near onset of instability: convection rolls form when the temperature difference between two plates overcomes the viscosity of the fluid in between. Basic patterns are typically periodic in, say, $x$, with a characteristic wavelength $L=2 \pi / k$. While such patterns can be readily analyzed mathematically using bifurcation theory, much less is known about imperfections in these perfect periodic patterns that arise in experiments and numerical simulations. Formally such imperfections or defects can be classified phenomenologically, leading to lists including defects such as grain boundaries, dislocations, and disclinations. Very little, however, is known about these defects beyond far-field approximations. Mere existence, for instance, is not known even for simple model approximations. Our goal here is to extend the methods used in [6] and show existence of grain boundaries near onset of instability. We remove a crucial restriction from [6] which forced the angle between grain boundaries to be larger than $\pi / 3$, effectively preventing interaction of the primary modes with other possible resonant modes. Our results confirm earlier findings in [8], where an approximation by amplitude equations was analyzed. We show that the approximation there, neglecting higher-order terms and non-adiabatic effects, as well as possible coupling to other, rotated modes, is justified. On the other hand, our results exclude to some extent, at small amplitude, bifurcations such as the ones observed in [2, 3].

Swift-Hohenberg equation We focus our attention on the arguably simplest pattern forming system, the Swift-Hohenberg equation

$$
\partial_{t} u=-(1+\Delta)^{2} u+\mu u-u^{3} .
$$

Here, $u(t, x, y)$ depends on $(x, y) \in \mathbb{R}^{2}$ and time $t \in \mathbb{R}$, and $\mu$ is a real parameter. Simple bifurcation analysis shows the existence of solutions $u_{\mathrm{r}}(k x ; k, \mu)$ which are spatially periodic $u_{\mathrm{r}}(\xi ; k, \mu)=u_{\mathrm{r}}(\xi+$ $2 \pi ; k, \mu)$, and even in $\xi$ for $\mu>0$, small. We refer to these stationary periodic patterns as roll solutions and denote rotated roll patterns as

$$
u_{\mathrm{r}}^{\varphi}(x, y ; k):=u_{\mathrm{r}}(k(x \cos \varphi-y \sin \varphi) ; k, \mu)
$$

with $\varphi \in[0,2 \pi)$.

Grain boundaries solutions Grain boundaries are solutions to (1.1) with $u_{t}=0$, that are asymptotic to roll solutions of different orientation as $x \rightarrow \pm \infty$. In the simplest case that we shall be interested in, here, they possess an additional reflection symmetry $x \mapsto-x$ and periodic in $y$. This can be seen as a maximal symmetry assumption for a grain boundary, since the pattern imposed by asymptotic roll solutions with different angles accomodates such a reflection symmetry and periodicity.

We construct the grain boundaries as solutions to the equation (1.1) which are steady and periodic in $y$ with wavenumber $k=k_{*}$ for sufficiently small $\mu$. Rescaling $y$, we need to solve

$$
0=-\left(1+\partial_{x}^{2}+k^{2} \partial_{y}^{2}\right)^{2} u+\mu u-u^{3}
$$

on $x, y \in \mathbb{R}^{2}$ with $2 \pi$-periodicity in $y$. 
Following [6], we also impose boundary conditions at infinity in the form

$$
\left|u_{\mathrm{gb}}\left(x-x_{ \pm}, y\right)-u_{\mathrm{r}}^{\varphi_{ \pm}}\left(x, y ; k_{ \pm}\right)\right| \rightarrow 0 \text { for } x \rightarrow \pm \infty .
$$

Here, the constants are suitable asymptotic phases $x_{ \pm}$, asymptotic angles $\varphi_{ \pm}$, and asymptotic wavenumbers $k_{ \pm}$for the roll solutions in the far field. Our symmetry assumption implies that $u_{\mathrm{gb}}(x, y)=$ $u_{\mathrm{gb}}(-x, y), \varphi_{-}=-\varphi_{+}, k_{+}=k_{-}$and $x_{+}=-x_{-}$. Possibly translating in $y$, we may assume that $x_{ \pm}=0$.

Main result Our main result states existence of such grain boundaries for small values of $\mu$ and arbitrary $\varphi_{ \pm}$.

Theorem 1 For every $\epsilon_{\varphi}>0$ there exists $\mu_{*}>0$ so that for every $\varphi_{-}-\varphi_{+}=\alpha \in\left(\epsilon_{\varphi}, \pi-\epsilon_{\varphi}\right)$, and any $0<\mu<\mu_{*}\left(\epsilon_{\varphi}\right)$, there exists a symmetric grain boundary between rolls of orientations $\varphi_{ \pm}$, for a selected wavenumber $k(\alpha, \mu)$.

We emphasize that the main difference from the results in [6] is that our theorem here covers arbitrary angles, removing the restriction to $\alpha>\pi / 3$. We also show existence for small $\mu$, uniformly in the angle $\alpha$. The limiting cases $\alpha=0$ and $\alpha=\pi$ are also interesting. Weak bending, $\alpha=\pi$, was studied in [7], basically showing existence in this regime as well. The limit $\alpha \rightarrow 0$ appears to be much more challenging. The remainder of this paper is occupied with the proof of Theorem 1. Our proof is based
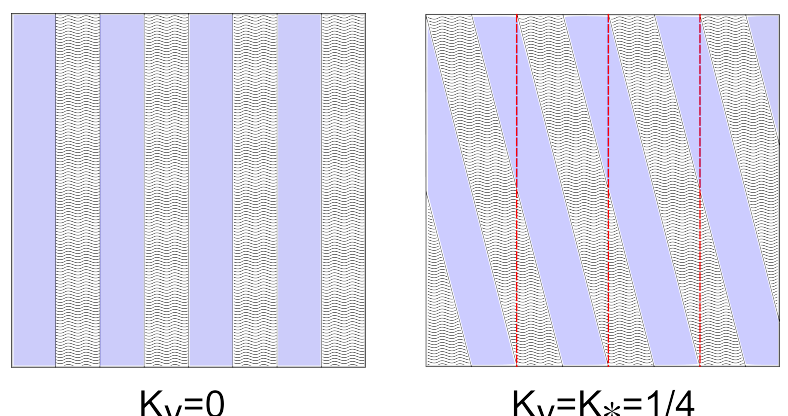

$\mathrm{K}_{\mathrm{y}}=\mathrm{K}_{*}=1 / 4$

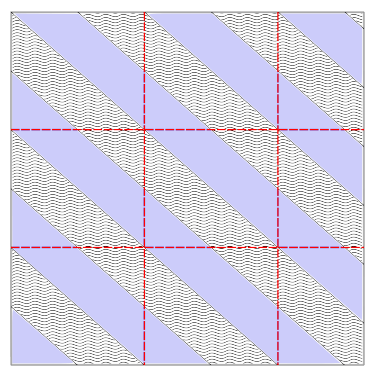

$$
\mathrm{K}_{\mathrm{y}}=3 \mathrm{~K}_{*}=3 / 4
$$

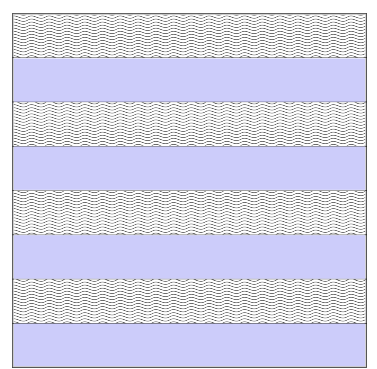

$$
\mathrm{K}_{\mathrm{y}}=4 \mathrm{~K}_{*}=1
$$

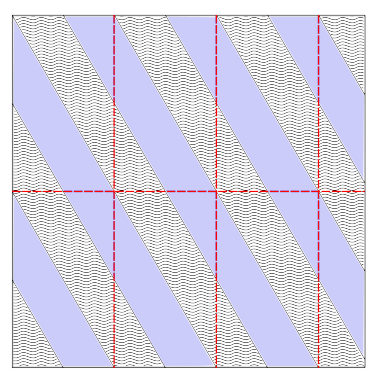

$\mathrm{K}_{\mathrm{y}}=2 \mathrm{~K}_{*}=1 / 2$

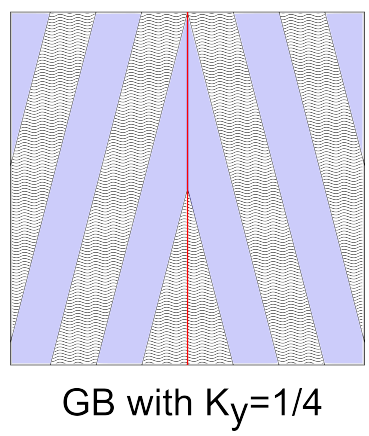

Figure 1.1: The pictures show compatible roll solutions and the grain boundary in the case $k_{*}=1 / 4$. The first five pictures show roll solutions with orientations comptible with the induced vertical $y$-period $8 \pi$. The red dashes show identical periodic cells in each case.

on spatial dynamics, rewriting (1.3) as an ill-posed dynamical system in the $x$-variable. We then follow 
the general strategy of bifurcation theory, using center-manifold reduction and normal form theory to identify a simple reduced differential equation. Within the leading-order terms of this equation, we find heteroclinic orbits that correspond to grain boundaries. We then proceed and show that these heteroclinic orbits persist under higher-order perturbations. We refer to [6] for more background on these methods and references to the literature.

While our methods build on the general strategy introduced in [6], there are several key differences. First, the dimension of the reduced ODE can be arbitrarily large, so that normal form theory is essential to reduce to a tractable system. The increase in dimension stems from the fact that for small $k_{*}$, that is, large $y$-period in the original scale, many orientations of roll solutions are compatible with periodicity in $y$. The analysis therefore needs to incorporate possible resonant interactions between the primary rolls and rotated linear and nonlinear modes. Major technical difficulties are introduced when rolls perpendicular to the grain boundary interface are compatible with $y$-periodicity. We refer to this case, $1 / k_{*} \in \mathbb{Z}$, as the resonant case. In fact, varying $k_{*}$ across such a resonance, the dimension of the center-manifold changes. The linearization exhibits a Jordan block of length 4, introducing a new scale into the system. As a consequence, we need a refined normal form theory. More crucially, the standard scaling $x \sim \mu^{-1 / 2}$ for the grain boundary introduces singularities in the perpendicular

mode, where length scales vary with $\mu^{-1 / 4}$. Moreover, as we vary $k_{*}$ near such a resonance, we find a subtle crossover between the $\mu^{-1 / 4}$ - and $\mu^{-1 / 2}$-scalings. When studying persistence, we then encounter a singularly perturbed differential operator in the linearization. We develop semi-explicit estimates on the Green's function for that operator and employ a frozen Newton method to show persistence.

Figure 1.1 illustrates some of these difficulties. The figure shows that, given $k_{*}$, there are possibly many different orientations of roll patterns that are compatible with the imposed $y$-periodicity. In the case shown there, $k_{*}=1 / 4$, there are 5 possible orientations. Note that the horizontal pattern is compatible only for integer choices of $1 / k_{*}$. As $k_{*}$ decreases past such resonances, more orientations become compatible with the periodicity in $y$. Note also that the figure shows how the grain boundaries we are finding possess maximal symmetry with respect to $y$-translations. In fact, we could have also tried to find grain boundaries between other orientations of rolls from Figure 1.1, which would possibly give non-symmetric grain boundaries, or grain boundaries with a $y$-period less than the imposed period. We will comment on these issues briefly, throughout the proofs.

Outline Our paper is organized as follows. Section 2 is concerned with the non-resonant case, $1 / k_{*} \notin \mathbb{Z}$. Section 3 treats resonance and near-resonance $1 / k_{*} \in \mathbb{Z}$.

\section{Grain boundaries for non-resonant angles $1 / k_{*} \notin \mathbb{Z}$}

We prove Theorem 1 in the non-resonant case. We first set up the spatial dynamics formulation, Section 2.1, then carry out the center-manifold reduction after identifying linearly neutral modes in Section 2.2. Normal form transformations put the system in a simple standard form, Section 2.3. We finally prove existence and persistence of heteroclinic orbits in Section 2.4. 


\subsection{Spatial dynamics}

Our basic approach follows [6]. We write the stationary Swift-Hohenberg equation (1.3) formally as a first-order differential equation in space $x$,

$$
\frac{d U}{d x}=\mathcal{A}(\mu, k) U+\mathcal{F}(U)
$$

setting

$$
U=\left(\begin{array}{c}
u \\
u_{1} \\
v \\
v_{1}
\end{array}\right), \quad \mathcal{A}(\mu, k)=\left(\begin{array}{cccc}
0 & 1 & 0 & 0 \\
-\left(1+k^{2} \partial_{y}^{2}\right) & 0 & 1 & 0 \\
0 & 0 & 0 & 1 \\
\mu & 0 & -\left(1+k^{2} \partial_{y}^{2}\right) & 0
\end{array}\right), \quad \mathcal{F}(U)=\left(\begin{array}{c}
0 \\
0 \\
0 \\
-u^{3}
\end{array}\right)
$$

While ill-posed as an initial-value problem, the operator $A(\mu, k)$ is bi-sectorial on the function space

$$
\mathcal{X}=H_{\mathrm{per}}^{3}(0,2 \pi) \times H_{\mathrm{per}}^{2}(0,2 \pi) \times H_{\mathrm{per}}^{1}(0,2 \pi) \times L^{2}(0,2 \pi),
$$

where

$$
H_{\mathrm{per}}^{j}(0,2 \pi)=\left\{u \in H_{\mathrm{loc}}^{j}(\mathbb{R}) ; u(z+2 \pi)=u(z), \forall z \in \mathbb{R}\right\}, \quad j \geqslant 1 .
$$

with domain

$$
\mathcal{D}(A)=\mathcal{Y}=H_{\text {per }}^{4}(0,2 \pi) \times H_{\text {per }}^{3}(0,2 \pi) \times H_{\text {per }}^{2}(0,2 \pi) \times H_{\text {per }}^{1}(0,2 \pi) .
$$

It is not difficult to verify that the nonlinear map $\mathcal{F}: \mathcal{Y} \rightarrow \mathcal{Y}$ is in fact smooth.

We are interested in solutions close to onset, that is, $\mu \sim 0, \mu>0$. We also allow for changes in the wavenumber, setting $k=k_{*}+\delta$ with $k_{*} \in(0,1)$ fixed and $\delta \sim 0$ small.

The choice of $k$ implicitly restricts angles and wavenumbers of bifurcating roll solutions. Since bifurcating rolls have wavenumber $\left(k_{x}, k_{y}\right)=\left(k_{0} \cos \varphi,-k_{0} \sin \varphi\right)$ with $k_{0} \sim 1$, the restriction to $2 \pi / k_{*^{-}}$ periodicity implies that $k_{0} \sin \varphi \in k_{*} \mathbb{Z}$. Equivalently, only angles

$$
\varphi=0, \pm \arcsin \left(k_{*}\right), \pm \arcsin \left(2 k_{*}\right), \ldots, \pm \arcsin \left(\left[\frac{1}{k_{*}}\right] k_{*}\right),
$$

where $[z]=\max \{m \in \mathbb{Z}, m \leqslant z\}$, are compatible with the periodic boundary conditions in $y$. Clearly, the number of compatible rolls increases when $k_{*}$ decreases and crosses through a resonance, that is, when $1 / k_{*}$ crosses an integer. We will see later that this fact is mirrored in a change in dimension of the center manifold. We will treat this resonant case later, in Section 3, and focus on the nonresonant case from now on. We point out that non-resonance precisely excludes rolls that are horizontal, that is, rolls perpendicular to the grain boundary interface, $\varphi=\pi / 2$.

\subsection{Center manifold reduction}

Central space. We view the system (2.1) as an infinite-dimensional dynamical system in the form

$$
\frac{d U}{d x}=\mathcal{A}_{*} U+\mathcal{B}(\mu, \delta) U+\mathcal{F}(U)
$$

with

$$
\mathcal{A}_{*}=\mathcal{A}\left(0, k_{*}\right), \quad \mathcal{B}(\mu, \delta)=\mathcal{A}\left(\mu, k_{*}+\delta\right)-\mathcal{A}\left(0, k_{*}\right)
$$


Noting that $\mathcal{A}_{*}: \mathcal{Y} \rightarrow \mathcal{X}$ is a continuous linear operator and $\mathcal{Y}$ is dense in and compactly embedded into $\mathcal{X}$, we conclude that the resolvent of $\mathcal{A}_{*}$ is compact and thus its spectrum $\sigma\left(\mathcal{A}_{*}\right)$ only consists of eigenvalues $\nu$. According to the dispersion relation,

$$
\nu^{2}=k_{*}^{2} \ell^{2}-1, \quad \ell \in \mathbb{Z}
$$

we have

$$
\sigma\left(\mathcal{A}_{*}\right)=\left\{\nu \in \mathbb{C} ; \nu^{2}=k_{*}^{2} \ell^{2}-1, \ell \in \mathbb{Z}\right\}
$$

Moreover,

$$
\sigma\left(\mathcal{A}_{*}\right) \cap \mathrm{i} \mathbb{R}=\left\{ \pm \mathrm{i} k_{\ell, x} \mid \ell \in I_{*}\right\}
$$

where

$$
k_{x, \ell}=\sqrt{1-\left(k_{*} \ell\right)^{2}}, \quad k_{x, \ell}=k_{x,-\ell}, \quad I_{*}=\left\{0, \pm 1, \ldots \pm \ell_{*}\right\}, \quad \ell_{*}=\left[\frac{1}{k_{*}}\right] .
$$

The non-resonance condition $1 / k_{*} \notin \mathbb{Z}$ implies that $k_{x, \ell} \neq 0$ for all $\ell$. Then it is not hard to see that the central space $\mathcal{X}_{c}$ of the operator $\mathcal{A}_{*}$, i.e., the spectral subspace associated with $\sigma\left(\mathcal{A}_{*}\right) \cap \mathrm{i} \mathbb{R}$, is $4\left(2 \ell_{*}+1\right)$-dimensional and spanned by the vectors $\left\{E_{\ell}, F_{\ell}, \overline{E_{\ell}}, \overline{F_{\ell}} \mid \ell \in I_{*}\right\}$, where, for every $\ell \in I_{*}$,

$$
E_{\ell}(y)=\left(\begin{array}{c}
1 \\
\mathrm{i} k_{x, \ell} \\
0 \\
0
\end{array}\right) \mathrm{e}^{\mathrm{i} \ell y}, \quad F_{\ell}(y)=\left(\begin{array}{c}
0 \\
1 \\
2 \mathrm{i} k_{x, \ell} \\
-2 k_{x, \ell}^{2}
\end{array}\right) \mathrm{e}^{\mathrm{i} \ell y}
$$

More specifically, the generalized eigenspace associated with the eigenvalue $\mathrm{i} k_{x, \ell}$ (or $-\mathrm{i} k_{x, \ell}$, respectively) is spanned by $E_{ \pm \ell}$ and $F_{ \pm \ell}$ (or $\overline{E_{ \pm \ell}}$ and $\overline{F_{ \pm \ell}}$, respectively), which satisfy

$$
\begin{gathered}
\mathcal{A}_{*} E_{ \pm \ell}=\mathrm{i} k_{x, \ell} E_{ \pm \ell}, \quad \mathcal{A}_{*} F_{ \pm \ell}=\mathrm{i} k_{x, \ell} F_{ \pm \ell}+E_{ \pm \ell}, \quad \text { for all } \ell \in I_{*}, \quad \text { and } \\
\mathcal{A}_{*} \overline{E_{ \pm \ell}}=-\mathrm{i} k_{x, \ell} \overline{E_{ \pm \ell}}, \quad \mathcal{A}_{*} \overline{F_{ \pm \ell}}=-\mathrm{i} k_{x, \ell} \overline{F_{ \pm \ell}}+\overline{E_{ \pm \ell}}, \quad \text { for all } \ell \in I_{*} .
\end{gathered}
$$

We point out here that while the eigenvalues $\pm \mathrm{i}$ are geometrically simple and algebraically double, the eigenvalues $\pm \mathrm{i} k_{x, \ell}$ are geometrically double and algebraically quadruple for $\ell \in I_{*} \backslash\{0\}$.

The next step is to calculate the spectral projection $\mathcal{P}_{c}: \mathcal{X} \rightarrow \mathcal{X}_{c}$. We first denote $\mathcal{A}_{*}^{\text {ad }}$ as the adjoint of $\mathcal{A}_{*}$ with respect to the scalar product $\langle\cdot, \cdot\rangle$ in $\left(L^{2}(0,2 \pi)\right)^{4}$ and

$$
\mathcal{A}_{*}^{\mathrm{ad}}=\left(\begin{array}{cccc}
0 & -\left(1+k_{*}^{2} \partial_{y}^{2}\right) & 0 & 0 \\
1 & 0 & 0 & 0 \\
0 & 1 & 0 & -\left(1+k_{*}^{2} \partial_{y}^{2}\right) \\
0 & 0 & 1 & 0
\end{array}\right)
$$

Similar to the $\mathcal{A}_{*}$ case, we have

$$
\mathcal{A}_{*}^{\mathrm{ad}} F_{\ell}^{\mathrm{ad}}=-\mathrm{i} k_{x, \ell} F_{\ell}^{\mathrm{ad}}, \quad \mathcal{A}_{*}^{\mathrm{ad}} E_{\ell}^{\mathrm{ad}}=-\mathrm{i} k_{x, \ell} E_{\ell}^{\mathrm{ad}}+F_{\ell}^{\mathrm{ad}}, \quad \ell \in I_{*},
$$

in which

$$
E_{\ell}^{\mathrm{ad}}(y)=\frac{1}{8 \pi k_{x, \ell}^{3}}\left(\begin{array}{c}
2 k_{x, \ell}^{3} \\
2 \mathrm{i} k_{x, \ell}^{2} \\
0 \\
\mathrm{i}
\end{array}\right) e^{\mathrm{i} \ell y}, \quad F_{\ell}^{\mathrm{ad}}(y)=\frac{1}{8 \pi k_{x, \ell}^{3}}\left(\begin{array}{c}
0 \\
0 \\
\mathrm{i} k_{x, \ell}^{2} \\
-k_{x, \ell}
\end{array}\right) e^{\mathrm{i} \ell y} \quad \ell \in I_{*} .
$$


Secondly, by definition, the spectral projection is given by

$$
\mathcal{P}_{c} U=\sum_{\ell \in I_{*}}\left(\left\langle U, E_{\ell}^{\mathrm{ad}}\right\rangle E_{\ell}+\left\langle U, F_{\ell}^{\mathrm{ad}}\right\rangle F_{\ell}+\left\langle U, \overline{E_{\ell}^{\mathrm{ad}}}\right\rangle \overline{E_{\ell}}+\left\langle U, \overline{F_{\ell}^{\mathrm{ad}}}\right\rangle \overline{F_{\ell}}\right)
$$

where

$$
\left\langle E_{\kappa}, E_{\ell}^{\mathrm{ad}}\right\rangle=\left\langle F_{\kappa}, F_{\ell}^{\mathrm{ad}}\right\rangle=\delta_{\kappa \ell}, \quad\left\langle F_{\kappa}, E_{\ell}^{\mathrm{ad}}\right\rangle=\left\langle E_{\kappa}, F_{\ell}^{\mathrm{ad}}\right\rangle=0, \quad \kappa, \ell \in I_{*} .
$$

Reduction to a center manifold. Through a center manifold reduction, we can reduce the PDE system to an ODE system while still keeping all small bounded solutions.

From (2.3)-(2.4), we note that the spectrum of $\left.\mathcal{A}_{*}\right|_{\left(\mathrm{id}-\mathcal{P}_{c}\right) \mathcal{X}}$ is off the imaginary axis. More precisely, we have, for some $\varepsilon>0$,

$$
\sigma\left(\left.\mathcal{A}_{*}\right|_{\left(\mathrm{id}-\mathcal{P}_{c}\right) \mathcal{X}}\right) \subset\{\lambda \in \mathbb{C} ;|\operatorname{Re} \lambda| \geqslant \varepsilon\},
$$

upon which, it is not hard to see that

$$
\left\|\left(\mathrm{i} \omega-\mathcal{A}_{*}\right)^{-1}\right\|_{\mathcal{L}\left(\left(\mathrm{id}-\mathcal{P}_{c}\right) \mathcal{X}\right)} \leqslant \frac{C}{1+|\omega|}, \quad \forall \omega \in \mathbb{R}
$$

with some positive constant $C>0$. Therefore, by applying the center manifold theorem( see $[4, \S 2])$, we can prove that there are neighborhoods of the origin $\mathcal{U} \subset \mathcal{X}_{c}, \mathcal{V} \subset\left(\right.$ id $\left.-\mathcal{P}_{c}\right) \mathcal{Y}, \mathcal{W} \subset \mathbb{R}^{2}$ and, for any $m<\infty$, a $C^{m}$-map $\boldsymbol{\Psi}: \mathcal{U} \times \mathcal{W} \rightarrow \mathcal{V}$ having the following properties.

(i) For any $(\mu, \delta) \in \mathcal{W}$, all bounded solutions $U(x)$ of $(2.2)$ within $\mathcal{U} \times \mathcal{V}$ are on the center manifold, i.e.,

$$
U(x)=U_{c}(x)+\Psi\left(U_{c}(x), \mu, \delta\right), \quad \forall x \in \mathbb{R} .
$$

(ii) The center manifold is tangent to the center eigenspace, i.e.,

$$
\left\|\Psi\left(U_{c}, \mu, \delta\right)\right\|_{\mathcal{Y}}=\mathcal{O}\left(|\mu|\left\|U_{c}\right\|+|\delta|\left\|U_{c}\right\|+\left\|U_{c}\right\|^{2}\right) .
$$

We now plug (2.5) into (2.2) and project it with $\mathcal{P}_{c}$, obtaining the reduced system for $U_{c}$,

$$
\frac{d U_{c}}{d x}=\mathcal{A}_{*} U_{c}+\mathcal{P}_{c}\left(\mathcal{B}(\mu, \delta) U_{c}+\mathcal{F}\left(U_{c}+\Psi\left(U_{c}, \mu, \delta\right)\right)\right)
$$

Remark 2.1 We have $\mathcal{P}_{c} \mathcal{B}(\mu, \delta) \Psi\left(U_{c}, \mu, \delta\right)=0$ due to the fact that

$$
\mathcal{X}_{c}=\mathcal{X}_{c}^{a d}:=\left\{\sum_{\ell \in I_{*}}\left(A_{\ell} E_{\ell}^{a d}+B_{\ell} F_{\ell}^{a d}+\overline{A_{\ell}} \overline{E_{\ell}^{a d}}+\overline{B_{\ell}} \overline{F_{\ell}^{a d}}\right) \mid A_{\ell} \in \mathbb{C}, B_{\ell} \in \mathbb{C}, \text { for all } \ell \in I_{*}\right\}
$$

and $B(\mu, \delta)$ maps $\left(\right.$ id $\left.-\mathcal{P}_{c}\right) \mathcal{Y}$ into $\left(\right.$ id $\left.-\mathcal{P}_{c}\right) \mathcal{X}$.

Lemma 2.2 For sufficiently small $|\delta|$, we have

$$
\left\|\Psi\left(U_{c}, \mu, \delta\right)\right\| \mathcal{Y}=\mathcal{O}\left(|\mu|\left\|U_{c}\right\|+\left\|U_{c}\right\|^{2}\right) .
$$


Proof. We need to show absence of terms of the form $\mathcal{O}\left(\delta\left|U_{c}\right|\right)$. For this, we notice that the tangent space to the center manifold is invariant under the linearization at any point of the center manifold. Since now the center manifold contains all equilibria, in particular the trivial equilibria $U=0, \delta$ small but possibly nonzero, we conclude that the tangent space to the center manifold at the origin, for all $\delta$ small, is given by the center eigenspace of the linearization at that equilibrium. The center eigenspace at the trivial equilibrium $U=0$ is in fact independent of $\delta$. To see this, note that any element in the center eigenspace can be represented in the form

$$
\sum_{\ell \in I_{*}}\left(A_{\ell} E_{\ell, \delta}+B_{\ell} F_{\ell, \delta}+\overline{A_{\ell} E_{\ell, \delta}}+\overline{B_{\ell} F_{\ell, \delta}}\right)
$$

where $A_{\ell}, B_{\ell}$ are complex-valued functions and

$$
E_{\ell, \delta}(y)=\left(\begin{array}{c}
1 \\
\mathrm{i} k_{x, \ell, \delta} \\
0 \\
0
\end{array}\right) \mathrm{e}^{\mathrm{i} \ell y}, \quad F_{\ell, \delta}(y)=\left(\begin{array}{c}
0 \\
1 \\
2 \mathrm{i} k_{x, \ell, \delta} \\
-2 k_{x, \ell, \delta}^{2,}
\end{array}\right) \mathrm{e}^{\mathrm{i} \ell y}
$$

Here, $k_{x, \ell, \delta}=\sqrt{1-\left(k_{*}+\delta\right)^{2} \ell^{2}}$. A direct computation shows that for $|\delta|$ sufficiently small, the central space

$$
\begin{aligned}
\mathcal{X}_{c, \delta} & :=\left\{\sum_{\ell \in I_{*}}\left(A_{\ell} E_{\ell, \delta}+B_{\ell} F_{\ell, \delta}+\overline{A_{\ell} E_{\ell, \delta}}+\overline{B_{\ell} F_{\ell, \delta}}\right) \mid A_{\ell}, B_{\ell} \in \mathbb{C}\right\} \\
& =\left\{\sum_{\ell \in I_{*}, \ell \geqslant 0}\left(R_{\ell} \cos (\ell y)+S_{\ell} \sin (\ell y)\right) \mid R_{\ell}, S_{\ell} \in \mathbb{R}^{4}\right\}
\end{aligned}
$$

is independent of the choice of $\delta$.

Reduced system. In this paragraph we calculate the Taylor jet of the vector field of our reduced system (2.6) up to order three. First of all, due to the estimate (2.7) and the fact that the nonlinearity $\mathcal{F}$ is cubic, a direct computation leads to

$$
\frac{d U_{c}}{d x}=\mathcal{A}_{*} U_{c}+\mathcal{P}_{c}\left(\mathcal{B}(\mu, \delta) U_{c}+\mathcal{F}\left(U_{c}\right)\right)+\mathcal{O}\left(|\mu|\left\|U_{c}\right\|^{3}+\left\|U_{c}\right\|^{4}\right) .
$$

Secondly, substituting

$$
U_{c}(x)=\sum_{\ell \in I_{*}}\left(A_{\ell}(x) E_{\ell}+B_{\ell}(x) F_{\ell}+\overline{A_{\ell}}(x) \overline{E_{\ell}}+\overline{B_{\ell}}(x) \overline{F_{\ell}}\right)
$$

into (2.8) lets us write the leading-order-term reduced system

$$
\frac{d U_{c}}{d x}=\mathcal{A}_{*} U_{c}+\mathcal{P}_{c}\left(\mathcal{B}(\mu) U_{c}+\mathcal{F}\left(U_{c}\right)\right)
$$

in terms of the basis $\left(A_{\ell}, B_{\ell}\right), \ell \in I_{*}$ as follows,

$$
\begin{aligned}
& A_{\ell}^{\prime}=\mathrm{i} k_{x, \ell} A_{\ell}+B_{\ell}-\mathrm{i} \frac{\mu+2 k_{x, \ell}^{2}\left(k_{x, \ell}^{2}-k_{x, \ell, \delta}^{2}\right)}{4 k_{x, \ell}^{3}} a_{\ell}+\frac{k_{x, \ell}^{2}-k_{x, \ell, \delta}^{2}}{2 k_{x, \ell}^{2}} b_{\ell}+\frac{\mathrm{i}}{4 k_{x, \ell}^{3}} P_{\ell}, \\
& B_{\ell}^{\prime}=\mathrm{i} k_{x, \ell} B_{\ell}-\frac{1}{4 k_{x, \ell}^{2}} \mu a_{\ell}-\mathrm{i} \frac{k_{x, \ell}^{2}-k_{x, \ell, \delta}^{2}}{2 k_{x, \ell}} b_{\ell}+\frac{1}{4 k_{x, \ell}^{2}} P_{\ell}
\end{aligned}
$$


in which

$$
\begin{array}{rlrl}
a_{\ell} & =A_{\ell}+\overline{A_{-\ell}}, \\
k_{x, \ell, \delta} & =\sqrt{1-\left(k_{*}+\delta\right)^{2} \ell^{2}}, \quad b_{\ell} & =B_{\ell}-\overline{B_{-\ell}}, \quad \text { for all } \ell \in I_{*}, \\
P_{\ell}\left(\left(A_{\ell}, \overline{A_{\ell}}\right)_{\ell \in I_{*}}\right) & =\sum_{\ell_{1}+\ell_{2}+\ell_{3}=\ell, \ell_{1}, \ell_{2}, \ell_{3} \in I_{*}} a_{\ell_{1}} a_{\ell_{2}} a_{\ell_{3}} .
\end{array}
$$

Symmetries. In the Swift-Hohenberg equation (1.3), there are three reflection symmetries

$$
y \mapsto-y, \quad x \mapsto-x, \quad u \mapsto-u,
$$

which are preserved all the way from the system (2.1) to the reduced system (2.6). For simplicity, we denote $I_{*}^{+}=\left\{0,1, \ldots, \ell_{*}\right\}$. The reflections $y \mapsto-y$ and $u \mapsto-u$ induce symmetries in the reduced system $(2.6)$

$$
\begin{aligned}
\mathcal{S}_{1}\left(A_{\ell}, B_{\ell}, \overline{A_{\ell}}, \overline{B_{\ell}}, A_{-\ell}, B_{-\ell}, \overline{A_{-\ell}}, \overline{B_{-\ell}}\right)^{T}=\left(A_{-\ell}, B_{-\ell}, \overline{A_{-\ell}}, \overline{B_{-\ell}}, A_{\ell}, B_{\ell}, \overline{A_{\ell}}, \overline{B_{\ell}}\right)^{T} \text {, for all } \ell \in I_{*}^{+} . \\
\mathcal{S}_{2}\left(A_{\ell}, B_{\ell}, \overline{A_{\ell}}, \overline{B_{\ell}}, A_{-\ell}, B_{-\ell}, \overline{A_{-\ell}}, \overline{B_{-\ell}}\right)^{T}=-\left(A_{\ell}, B_{\ell}, \overline{A_{\ell}}, \overline{B_{\ell}}, A_{-\ell}, B_{-\ell}, \overline{A_{-\ell}}, \overline{B_{-\ell}}\right)^{T} \text {, for all } \ell \in I_{*}^{+} .
\end{aligned}
$$

The reflection $x \mapsto-x$ implies that the reduced vector field (2.6) anticommutes with

$$
\mathcal{R}\left(A_{\ell}, B_{\ell}, \overline{A_{\ell}}, \overline{B_{\ell}}, A_{-\ell}, B_{-\ell}, \overline{A_{-\ell}}, \overline{B_{-\ell}}\right)^{T}=\left(\overline{A_{-\ell}},-\overline{B_{-\ell}}, A_{-\ell},-B_{-\ell}, \overline{A_{\ell}},-\overline{B_{\ell}}, A_{\ell},-B_{\ell}\right)^{T} \text {, for all } \ell \in I_{*}^{+} .
$$

Therefore, there are no even order $U_{c}$-terms in the reduced system (2.8), that is,

$$
\frac{d U_{c}}{d x}=\mathcal{A}_{*} U_{c}+\mathcal{P}_{c}\left(\mathcal{B}(\mu, \delta) U_{c}+\mathcal{F}\left(U_{c}\right)\right)+\mathcal{O}\left(|\mu|\left\|U_{c}\right\|^{3}+\left\|U_{c}\right\|^{5}\right) .
$$

Moreover, the invariance of the Swift-Hohenberg equation (1.3) under translations in $y$ induces the symmetry for all $\ell \in I_{*}^{+}$,

$$
\begin{gathered}
\mathcal{T}_{\phi}\left(A_{\ell}, B_{\ell}, \overline{A_{\ell}}, \overline{B_{\ell}}, A_{-\ell}, B_{-\ell}, \overline{A_{-\ell}}, \overline{B_{-\ell}}\right)^{T}= \\
\left(\mathrm{e}^{i \ell \phi} A_{\ell}, \mathrm{e}^{i \ell \phi} B_{\ell}, \mathrm{e}^{-i \ell \phi} \overline{A_{\ell}}, \mathrm{e}^{-i \ell \phi} \overline{B_{\ell}}, \mathrm{e}^{-i \ell \phi} A_{-\ell}, \mathrm{e}^{-i \ell \phi} B_{-\ell}, \mathrm{e}^{i \ell \phi} \overline{A_{-\ell}}, \mathrm{e}^{i \ell \phi} \overline{B_{-\ell}}\right)^{T} .
\end{gathered}
$$

Remark 2.3 For the case $\ell=0$, the formulas above are somewhat overly complicated since then, of course, $A_{\ell}=A_{-\ell}$, etc. Still, the formulas hold true in this case as well.

\subsection{Normal form transformations}

In this section, a composition of linear and nonlinear transformations leads to a normal form of the reduced system (2.10). We first simplify the linear terms in (2.9) and then simplify the cubic terms.

Versal linear transformation. Consider the linear part of the system (2.9), for $\ell \in I_{*}$,

$$
\begin{aligned}
& A_{\ell}^{\prime}=\mathrm{i} k_{x, \ell} A_{\ell}+B_{\ell}-\mathrm{i} \frac{\mu+2 k_{x, \ell}^{2}\left(k_{x, \ell}^{2}-k_{x, \ell, \delta}^{2}\right)}{4 k_{x, \ell}^{3}} a_{\ell}+\frac{k_{x, \ell}^{2}-k_{x, \ell, \delta}^{2}}{2 k_{x, \ell}^{2}} b_{\ell} \\
& B_{\ell}^{\prime}=\mathrm{i} k_{x, \ell} B_{\ell}-\frac{1}{4 k_{x, \ell}^{2}} \mu a_{\ell}-\mathrm{i} \frac{k_{x, \ell}^{2}-k_{x, \ell, \delta}^{2}}{2 k_{x, \ell}} b_{\ell} .
\end{aligned}
$$


Lemma 2.4 For all $\ell \in I_{*}^{+}$, there exist smooth linear maps $L_{\ell}(\mu, \delta)$ such that, for sufficiently small $\mu$ and $\delta$, the linear changes of variables,

$$
\left(A_{\ell}, B_{\ell}, \overline{A_{\ell}}, \overline{B_{\ell}}, A_{-\ell}, B_{-\ell}, \overline{A_{-\ell}}, \overline{B_{-\ell}}\right)^{T}=L_{\ell}(\mu, \delta)\left(C_{\ell}, D_{\ell}, \overline{C_{\ell}}, \overline{D_{\ell}}, C_{-\ell}, D_{-\ell}, \overline{C_{-\ell}}, \overline{D_{-\ell}}\right)^{T}
$$

transform the system (2.11) into the normal form

$$
\begin{aligned}
C_{\ell}^{\prime} & =\mathrm{i} \sqrt{\frac{1}{2}\left(k_{x, \ell, \delta}^{2}+\sqrt{k_{x, \ell, \delta}^{4}-\mu}\right)} C_{\ell}+D_{\ell} \\
D_{\ell}^{\prime} & =-\frac{1}{2}\left(k_{x, \ell, \delta}^{2}-\sqrt{k_{x, \ell, \delta}^{4}-\mu}\right) C_{\ell}+\mathrm{i} \sqrt{\frac{1}{2}\left(k_{x, \ell, \delta}^{2}+\sqrt{k_{x, \ell, \delta}^{4}-\mu}\right)} D_{\ell} .
\end{aligned}
$$

Moreover, we have the following properties for $L_{\ell}(\mu, \delta)$ :

- $L_{\ell}(\mu, \delta)$ commutes with the symmetries $\mathcal{S}_{1}, \mathcal{S}_{2}, \mathcal{R}$, and $\mathcal{T}_{\phi}, \phi \in \mathbb{R}$;

- $L_{\ell}(0,0)=\mathrm{id}$ and, we can choose

$$
L_{\ell}(0, \delta)=\left(\begin{array}{cccccccc}
\alpha_{1} & \mathrm{i} \alpha_{3} & 0 & 0 & 0 & 0 & \alpha_{4} & \mathrm{i} \alpha_{3} \\
0 & \alpha_{2} & 0 & 0 & 0 & 0 & 0 & \alpha_{5} \\
0 & 0 & \alpha_{1} & -\mathrm{i} \alpha_{3} & \alpha_{4} & -\mathrm{i} \alpha_{3} & 0 & 0 \\
0 & 0 & 0 & \alpha_{2} & 0 & \alpha_{5} & 0 & 0 \\
0 & 0 & \alpha_{4} & \mathrm{i} \alpha_{3} & \alpha_{1} & \mathrm{i} \alpha_{3} & 0 & 0 \\
0 & 0 & 0 & \alpha_{5} & 0 & \alpha_{2} & 0 & 0 \\
\alpha_{4} & -\mathrm{i} \alpha_{3} & 0 & 0 & 0 & 0 & \alpha_{1} & -\mathrm{i} \alpha_{3} \\
0 & \alpha_{5} & 0 & 0 & 0 & 0 & 0 & \alpha_{2}
\end{array}\right),
$$

where

$$
\begin{array}{r}
\alpha_{1}=\frac{k_{x, \ell, \delta}+k_{x, \ell}}{2 k_{x, \ell}}, \quad \alpha_{2}=\frac{k_{x, \ell, \delta}\left(k_{x, \ell, \delta}+k_{x, \ell}\right)}{2 k_{x, \ell}^{2}}, \quad \alpha_{3}=\frac{k_{x, \ell, \delta}^{2}-k_{x, \ell}^{2}}{2 k_{x, \ell}^{3}}, \\
\alpha_{4}=-\frac{k_{x, \ell, \delta}-k_{x, \ell}}{k_{x, \ell}}, \quad \alpha_{5}=\frac{k_{x, \ell, \delta}\left(k_{x, \ell, \delta}-k_{x, \ell}\right)}{2 k_{x, \ell}^{2}} .
\end{array}
$$

Proof. We will apply Theorem 4.4 in [1] to simplify the linear part. To do that, we rewrite the systems (2.11) and (2.13) separately as follows. For all $\ell \in I_{*}^{+}$,

$$
\begin{aligned}
\frac{\mathrm{d}}{\mathrm{d} x}\left(A_{\ell}, B_{\ell}, \overline{A_{\ell}}, \overline{B_{\ell}}, A_{-\ell}, B_{-\ell}, \overline{A_{-\ell}}, \overline{B_{-\ell}}\right)^{T} & =M_{\ell}(\mu, \delta)\left(A_{\ell}, B_{\ell}, \overline{A_{\ell}}, \overline{B_{\ell}}, A_{-\ell}, B_{-\ell}, \overline{A_{-\ell}}, \overline{B_{-\ell}}\right)^{T}, \\
\frac{\mathrm{d}}{\mathrm{d} x}\left(C_{\ell}, D_{\ell}, \overline{C_{\ell}}, \overline{D_{\ell}}, C_{-\ell}, D_{-\ell}, \overline{C_{-\ell}}, \overline{D_{-\ell}}\right)^{T} & =N_{\ell}(\mu, \delta)\left(C_{\ell}, D_{\ell}, \overline{C_{\ell}}, \overline{D_{\ell}}, C_{-\ell}, D_{-\ell}, \overline{C_{-\ell}}, \overline{D_{-\ell}}\right)^{T} .
\end{aligned}
$$

By the symmetries of these matrices, we conclude that $M_{\ell}(\mu, \delta), N_{\ell}(\mu, \delta) \in \mathcal{M}$, where

$$
\mathcal{M}:=\left\{M \in \mathrm{M}^{8 \times 8} \mid M \mathcal{S}_{j}=\mathcal{S}_{j} M, M \mathcal{R}=-\mathcal{R} M, M \mathcal{T}_{\phi}=\mathcal{T}_{\phi} M, \text { for all } j=1,2 \text {, and } \phi \in \mathbb{R}\right\} .
$$


Now the key part is to find a versal deformation of $M_{\ell}(0,0)$ in $\mathcal{M}$. On one hand, we note that

$$
M_{\ell}(0,0)=\left(\begin{array}{cccccccc}
\mathrm{i} k_{x, \ell} & 1 & 0 & 0 & 0 & 0 & 0 & 0 \\
0 & \mathrm{i} k_{x, \ell} & 0 & 0 & 0 & 0 & 0 & 0 \\
0 & 0 & -\mathrm{i} k_{x, \ell} & 1 & 0 & 0 & 0 & 0 \\
0 & 0 & 0 & -\mathrm{i} k_{x, \ell} & 0 & 0 & 0 & 0 \\
0 & 0 & 0 & 0 & \mathrm{i} k_{x, \ell} & 1 & 0 & 0 \\
0 & 0 & 0 & 0 & 0 & \mathrm{i} k_{x, \ell} & 0 & 0 \\
0 & 0 & 0 & 0 & 0 & 0 & -\mathrm{i} k_{x, \ell} & 1 \\
0 & 0 & 0 & 0 & 0 & 0 & 0 & -\mathrm{i} k_{x, \ell}
\end{array}\right)
$$

is already in its Jordan form. The commutator of $M_{\ell}^{*}(0,0)$ in $\mathcal{M}$ consists of matrices of the general form

$$
\widetilde{N}=\left(\begin{array}{cccccccc}
\mathrm{i} \gamma_{1} & 0 & 0 & 0 & 0 & 0 & 0 & 0 \\
\gamma_{2} & \mathrm{i} \gamma_{1} & 0 & 0 & 0 & 0 & 0 & 0 \\
0 & 0 & -\mathrm{i} \gamma_{1} & 0 & 0 & 0 & 0 & 0 \\
0 & 0 & \gamma_{2} & -\mathrm{i} \gamma_{1} & 0 & 0 & 0 & 0 \\
0 & 0 & 0 & 0 & \mathrm{i} \gamma_{1} & 0 & 0 & 0 \\
0 & 0 & 0 & 0 & \gamma_{2} & \mathrm{i} \gamma_{1} & 0 & 0 \\
0 & 0 & 0 & 0 & 0 & 0 & -\mathrm{i} \gamma_{1} & 0 \\
0 & 0 & 0 & 0 & 0 & 0 & \gamma_{2} & -\mathrm{i} \gamma_{1}
\end{array}\right)
$$

where $\gamma_{1 \backslash 2} \in \mathbb{R}$. Therefore, $M_{\ell}(0,0)+\tilde{N}\left(\gamma_{1}, \gamma_{2}\right)$ is a versal deformation of $M_{\ell}(0,0)$. Moreover, by Theorem 4.4 in [1], there exists a smooth deformation of the identity matrix, denoted as $L_{\ell}(\mu, \delta)$ and two smooth functions $\gamma_{1 \backslash 2}(\mu, \delta)$ such that, for sufficiently small $\mu$ and $\delta$,

$$
M_{\ell}(\mu, \delta)=L_{\ell}(\mu, \delta)\left(M_{\ell}(0,0)+\widetilde{N}\left(\gamma_{1}(\mu, \delta), \gamma_{2}(\mu, \delta)\right)\right) L_{\ell}^{-1}(\mu, \delta)
$$

From the original system, it is straightforward to see that the characteristic polynomial $P(\lambda)$ of $M_{\ell}(\mu, \delta)$ is

$$
P(\lambda)=\left(\mu-\left(1+\lambda^{2}-\left(k_{*}+\delta\right)^{2} \ell^{2}\right)^{2}\right)^{2}
$$

On the other hand,

$$
P(\lambda)=\operatorname{det}\left(\lambda-M_{\ell}(0,0)-\tilde{N}\right)=\left(\left(\lambda^{2}+\left(k_{x, \ell}+\gamma_{1}\right)^{2}\right)^{2}-2 \gamma_{2}\left(\lambda^{2}-\left(k_{x, \ell}+\gamma_{1}\right)^{2}\right)+\gamma_{2}^{2}\right)^{2} .
$$

Comparing the coefficients of the above two polynomials, we have

$$
\begin{aligned}
\gamma_{1}+k_{x, \ell} & =\sqrt{\frac{1}{2}\left(k_{x, \ell, \delta}^{2}+\sqrt{k_{x, \ell, \delta}^{4}-\mu}\right)} \\
\gamma_{2} & =-\frac{1}{2}\left(k_{x, \ell, \delta}^{2}-\sqrt{k_{x, \ell, \delta}^{4}-\mu}\right) .
\end{aligned}
$$

Moreover, $N_{\ell}(\mu, \delta)=M_{\ell}(0,0)+\tilde{N}\left(\gamma_{1}(\mu, \delta), \gamma_{2}(\mu, \delta)\right)$. As for the choice of $L_{\ell}(0, \delta)$, we just note that $M_{\ell}(0, \delta)$ and $N_{\ell}(0, \delta)$ are the linear systems separately under two sets of bases $\left\{E_{\ell}, F_{\ell}\right\}_{\ell \in I_{*}}$ and $\left\{E_{\ell, \delta}, F_{\ell, \delta}\right\}_{\ell \in I_{*}}$. Thus, $L_{\ell}(0, \delta)$ is chosen naturally as the corresponding transition matrix. 
Based on Lemma 2.4, our reduced system (2.9)-(2.10) has the following expression. For all $\ell \in I_{*}$,

$$
\begin{aligned}
C_{\ell}^{\prime} & =\mathrm{i} \sqrt{\frac{1}{2}\left(k_{x, \ell, \delta}^{2}+\sqrt{k_{x, \ell, \delta}^{4}-\mu}\right)} C_{\ell}+D_{\ell}+\frac{\mathrm{i}}{4 k_{x, \ell, \delta}^{3}} P_{\ell} \\
D_{\ell}^{\prime} & =-\frac{1}{2}\left(k_{x, \ell, \delta}^{2}-\sqrt{k_{x, \ell, \delta}^{4}-\mu}\right) C_{\ell}+\mathrm{i} \sqrt{\frac{1}{2}\left(k_{x, \ell, \delta}^{2}+\sqrt{k_{x, \ell, \delta}^{4}-\mu}\right)} D_{\ell}+\frac{1}{4 k_{x, \ell, \delta}^{2}} P_{\ell},
\end{aligned}
$$

with higher order terms in the form

$$
\mathcal{O}\left(|\mu|\left(\sum_{\ell \in I_{*}}\left|C_{\ell}\right|+\left|D_{\ell}\right|\right)^{3}+\left(\sum_{\ell \in I_{*}}\left|C_{\ell}\right|+\left|D_{\ell}\right|\right)^{5}\right) .
$$

Cubic transformation. Now we consider the leading order of system (2.15) for $\mu=0$ and $\ell \in I_{*}$,

$$
\begin{aligned}
A_{\ell}^{\prime} & =\mathrm{i} k_{x, \ell, \delta} A_{\ell}+B_{\ell}+\frac{\mathrm{i}}{4 k_{x, \ell, \delta}^{3}} P_{\ell} \\
B_{\ell}^{\prime} & =\mathrm{i} k_{x, \ell, \delta} B_{\ell}+\frac{1}{4 k_{x, \ell, \delta}^{2}} P_{\ell} .
\end{aligned}
$$

In order to find a normal form of (2.16), we first prove the following lemma.

Lemma 2.5 For $z_{i} \in \mathbb{C},\left|z_{i}\right|=1, i=1,2,3,4$, we have $z_{1}+z_{2}+z_{3}+z_{4}=0$ if and only if $z_{1}=-z_{2}$ and $z_{3}=-z_{4}$, possibly after permuting the indices.

Proof. The lemma is a formulation of the somewhat folklore fact that the only equilateral quadrilateral is the rhombus. In fact, the "if" part is clear. To prove the "only if" part, we interpret the $z_{j}$ as vectors, so that the equation $z_{1}+z_{2}+z_{3}+z_{4}=0$ corresponds to the fact that the vectors form a quadrilateral when attached at endpoints. Now intersecting the quadrilateral with a diagonal, we obtain two congruent triangles since sides have equal lengths. This implies that the equilateral quadrilateral is a parallelogram and thus a rhombus.

Lemma 2.6 For all $\ell \in I_{*}$, there exist smooth families of homogeneous polynomials $\left\{\Phi_{\ell}(\delta), \Psi_{\ell}(\delta)\right\}_{\ell \in I_{*}}$ of degree 3 in the complex variables $\left(C_{\kappa}, D_{\kappa}, \overline{C_{\kappa}}, \overline{D_{\kappa}}\right)_{\kappa \in I_{*}}$, such that the change of variables

$$
\begin{aligned}
& A_{\ell}=C_{\ell}+\Phi_{\ell}(\delta)\left(\left(C_{\kappa}, D_{\kappa}, \overline{C_{\kappa}}, \overline{D_{\kappa}}\right)_{\kappa \in I_{*}}\right), \\
& B_{\ell}=D_{\ell}+\Psi_{\ell}(\delta)\left(\left(C_{\kappa}, D_{\kappa}, \overline{C_{\kappa}}, \overline{D_{\kappa}}\right)_{\kappa \in I_{*}}\right),
\end{aligned}
$$

is well-defined in a neighborhood of the origin and transforms the system (2.16) into the normal form

$$
\begin{aligned}
C_{\ell}^{\prime}= & \mathrm{i} k_{x, \ell, \delta} C_{\ell}+D_{\ell}+\mathcal{O}\left(\left(\sum_{\kappa \in I_{*}}\left|C_{\kappa}\right|+\left|D_{\kappa}\right|\right)^{5}\right) \\
D_{\ell}^{\prime}= & \mathrm{i} k_{x, \ell, \delta} D_{\ell}+\frac{3}{4 k_{x, \ell, \delta}^{2}} C_{\ell}\left(-\left|C_{\ell}\right|^{2}+2 \sum_{\kappa \in I_{*}}\left|C_{\kappa}\right|^{2}\right)+\frac{3 \mathrm{i}}{4 k_{x, \ell, \delta}^{3}} D_{\ell}\left(-\left|C_{\ell}\right|^{2}+2 \sum_{\kappa \in I_{*}}\left|C_{\kappa}\right|^{2}\right) \\
& +\frac{3 \mathrm{i}}{4 k_{x, \ell, \delta}^{3}} C_{\ell}\left(-\left(C_{\ell} \overline{D_{\ell}}+\overline{C_{\ell}} D_{\ell}\right)+2 \sum_{\kappa \in I_{*}}\left(C_{\kappa} \overline{D_{\kappa}}+\overline{C_{\kappa}} D_{\kappa}\right)\right) \\
& +\mathcal{O}\left(\left(\sum_{\kappa \in I_{*}}\left|C_{\kappa}\right|+\left|D_{\kappa}\right|\right)^{5}\right)
\end{aligned}
$$




\section{Proof.}

Denote the cubic terms in the right hand sides of the systems $(2.16)$ and $(2.18)$ by $\left(F_{\ell}, G_{\ell}\right)_{\ell \in I_{*}}$ and $\left(0, N_{\ell}\right)_{\ell \in I_{*}}$, respectively. Following the proof of the normal form theorem (see for instance [4, $\left.\S 3\right]$ ), substituting (2.17) into (2.16), and taking into account (2.18) at order 3 in the resulting equalities we find that the polynomials $\left\{\Phi_{\ell}, \Psi_{\ell}\right\}_{\ell \in I_{*}}$ satisfy the equations, for all $\ell \in I_{*}$,

$$
\left(\mathcal{D}-\mathrm{i} k_{x, \ell, \delta}\right) \Phi_{\ell}=\Psi_{\ell}+F_{\ell}, \quad\left(\mathcal{D}-\mathrm{i} k_{x, \ell, \delta}\right) \Psi_{\ell}=G_{\ell}-N_{\ell}
$$

in which

$$
\mathcal{D}=\sum_{\ell \in I_{*}}\left(\left(\mathrm{i} k_{x, \ell, \delta} C_{\ell}+D_{\ell}\right) \frac{\partial}{\partial C_{\ell}}+\mathrm{i} k_{x, \ell, \delta} D_{\ell} \frac{\partial}{\partial D_{\ell}}+\left(-\mathrm{i} k_{x, \ell, \delta} \overline{C_{\ell}}+\overline{D_{\ell}}\right) \frac{\partial}{\partial \overline{C_{\ell}}}+\left(-\mathrm{i} k_{x, \ell, \delta} \overline{D_{\ell}}\right) \frac{\partial}{\partial \overline{D_{\ell}}}\right) .
$$

The polynomials $\Phi_{\ell}, \Psi_{\ell}$ exist provided the right hand sides in the equations (2.19) belong to the range of $\mathcal{D}-\mathrm{i} k_{x, \ell, \delta}$.

We have

$$
F_{\ell}=\frac{\mathrm{i}}{4 k_{x, \ell, \delta}^{3}} P_{\ell}, \quad G_{\ell}=\frac{1}{4 k_{x, \ell, \delta}^{2}} P_{\ell}
$$

with

$$
P_{\ell}\left(\left(C_{\ell}, \overline{C_{\ell}}\right)_{\ell \in I_{*}}\right)=\sum_{\substack{\ell_{1}+\ell_{2}+\ell_{3}=\ell, \ell_{1}, \ell_{2}, \ell_{3} \in I_{*}}} c_{\ell_{1}} c_{\ell_{2}} c_{\ell_{3}}, \quad c_{\ell}=C_{\ell}+\overline{C_{-\ell}}, \quad \overline{c_{\ell}}=c_{-\ell}, \quad \text { for all } \ell \in I_{*} .
$$

In order to see which monomials in $P_{\ell}$ are in the range of $\mathcal{D}-\mathrm{i} k_{x, \ell}$, we first notice that

$$
\begin{aligned}
c_{\ell_{1}} c_{\ell_{2}} c_{\ell_{3}}= & \left(C_{\ell_{1}}+\overline{C_{-\ell_{1}}}\right)\left(C_{\ell_{2}}+\overline{C_{-\ell_{2}}}\right)\left(C_{\ell_{3}}+\overline{C_{-\ell_{3}}}\right) \\
= & C_{\ell_{1}} C_{\ell_{2}} C_{\ell_{3}}+\left(C_{\ell_{1}} C_{\ell_{2}} \overline{C_{-\ell_{3}}}+C_{\ell_{1}} \overline{C_{-\ell_{2}}} C_{\ell_{3}}+\overline{C_{-\ell_{1}}} C_{\ell_{2}} C_{\ell_{3}}\right) \\
& +\left(C_{\ell_{1}} \overline{C_{-\ell_{2}} C_{-\ell_{3}}}+\overline{C_{-\ell_{1}} C_{-\ell_{2}}} C_{\ell_{3}}+\overline{C_{-\ell_{1}}} C_{\ell_{2}} \overline{C_{-\ell_{3}}}\right)+\overline{C_{-\ell_{1}} C_{-\ell_{2}} C_{-\ell_{3}}},
\end{aligned}
$$

which indicates that we have essentially only 4 types of monomials regardless of permutations

$$
C_{\ell_{1}} C_{\ell_{2}} C_{\ell_{3}}, \quad C_{\ell_{1}} C_{\ell_{2}} \overline{C_{-\ell_{3}}}, \quad C_{\ell_{1}} \overline{C_{-\ell_{2}} C_{-\ell_{3}}}, \quad \overline{C_{-\ell_{1}} C_{-\ell_{2}} C_{-\ell_{3}}} .
$$

We discuss all possibly cases, next.

- Monomials of type " $C_{\ell_{1}} C_{\ell_{2}} C_{\ell_{3}}$ " are in the range of $\mathcal{D}-\mathrm{i} k_{x, \ell}$. First, we notice that

$$
\begin{aligned}
\left(\mathcal{D}-\mathrm{i} k_{x, \ell, \delta}\right) C_{\ell_{1}} C_{\ell_{2}} C_{\ell_{3}}= & \mathrm{i}\left(k_{x, \ell_{1}, \delta}+k_{x, \ell_{2}, \delta}+k_{x, \ell_{3}, \delta}-k_{x, \ell, \delta}\right) C_{\ell_{1}} C_{\ell_{2}} C_{\ell_{3}} \\
& +D_{\ell_{1}} C_{\ell_{2}} C_{\ell_{3}}+C_{\ell_{1}} D_{\ell_{2}} C_{\ell_{3}}+C_{\ell_{1}} C_{\ell_{2}} D_{\ell_{3}}, \\
\left(\mathcal{D}-\mathrm{i} k_{x, \ell, \delta}\right) D_{\ell_{1}} C_{\ell_{2}} C_{\ell_{3}}= & \mathrm{i}\left(k_{x, \ell_{1}, \delta}+k_{x, \ell_{2}, \delta}+k_{x, \ell_{3}, \delta}-k_{x, \ell, \delta}\right) D_{\ell_{1}} C_{\ell_{2}} C_{\ell_{3}} \\
& +D_{\ell_{1}} D_{\ell_{2}} C_{\ell_{3}}+D_{\ell_{1}} C_{\ell_{2}} D_{\ell_{3}}, \\
\left(\mathcal{D}-\mathrm{i} k_{x, \ell, \delta}\right) C_{\ell_{1}} D_{\ell_{2}} C_{\ell_{3}}= & \mathrm{i}\left(k_{x, \ell_{1}, \delta}+k_{x, \ell_{2}, \delta}+k_{x, \ell_{3}, \delta}-k_{x, \ell, \delta}\right) C_{\ell_{1}} D_{\ell_{2}} C_{\ell_{3}} \\
& +D_{\ell_{1}} D_{\ell_{2}} C_{\ell_{3}}+C_{\ell_{1}} D_{\ell_{2}} D_{\ell_{3}}, \\
\left(\mathcal{D}-\mathrm{i} k_{x, \ell, \delta}\right) C_{\ell_{1}} C_{\ell_{2}} D_{\ell_{3}}= & \mathrm{i}\left(k_{x, \ell_{1}, \delta}+k_{x, \ell_{2}, \delta}+k_{x, \ell_{3}, \delta}-k_{x, \ell, \delta}\right) C_{\ell_{1}} C_{\ell_{2}} D_{\ell_{3}} \\
& +D_{\ell_{1}} C_{\ell_{2}} D_{\ell_{3}}+C_{\ell_{1}} D_{\ell_{2}} D_{\ell_{3}},
\end{aligned}
$$




$$
\begin{aligned}
\left(\mathcal{D}-\mathrm{i} k_{x, \ell, \delta}\right) D_{\ell_{1}} D_{\ell_{2}} C_{\ell_{3}}= & \mathrm{i}\left(k_{x, \ell_{1}, \delta}+k_{x, \ell_{2}, \delta}+k_{x, \ell_{3}, \delta}-k_{x, \ell, \delta}\right) D_{\ell_{1}} D_{\ell_{2}} C_{\ell_{3}} \\
& +D_{\ell_{1}} D_{\ell_{2}} D_{\ell_{3}}, \\
\left(\mathcal{D}-\mathrm{i} k_{x, \ell, \delta}\right) D_{\ell_{1}} C_{\ell_{2}} D_{\ell_{3}}= & \mathrm{i}\left(k_{x, \ell_{1}, \delta}+k_{x, \ell_{2}, \delta}+k_{x, \ell_{3}, \delta}-k_{x, \ell, \delta}\right) D_{\ell_{1}} C_{\ell_{2}} D_{\ell_{3}}+D_{\ell_{1}} D_{\ell_{2}} D_{\ell_{3}}, \\
\left(\mathcal{D}-\mathrm{i} k_{x, \ell, \delta}\right) C_{\ell_{1}} D_{\ell_{2}} D_{\ell_{3}}= & \mathrm{i}\left(k_{x, \ell_{1}, \delta}+k_{x, \ell_{2}, \delta}+k_{x, \ell_{3}, \delta}-k_{x, \ell, \delta}\right) C_{\ell_{1}} D_{\ell_{2}} D_{\ell_{3}}+D_{\ell_{1}} D_{\ell_{2}} D_{\ell_{3}}, \\
\left(\mathcal{D}-\mathrm{i} k_{x, \ell, \delta}\right) D_{\ell_{1}} D_{\ell_{2}} D_{\ell_{3}}= & \mathrm{i}\left(k_{x, \ell_{1}, \delta}+k_{x, \ell_{2}, \delta}+k_{x, \ell_{3}, \delta}-k_{x, \ell, \delta}\right) D_{\ell_{1}} D_{\ell_{2}} D_{\ell_{3}} .
\end{aligned}
$$

Moreover, according to lemma 2.5 and the fact that

$$
\left\{\begin{array}{l}
\left|k_{x, \ell_{j}, \delta}+i\left(k_{*}+\delta\right) \ell_{j}\right|=1, \quad j=1,2,3 \\
\left|k_{x, \ell, \delta}+i\left(k_{*}+\delta\right) \ell\right|=1 \\
\ell_{1}+\ell_{2}+\ell_{3}-\ell=0 \\
k_{x, \ell, \delta}, k_{x, \ell_{j}, \delta}>0, \quad j=1,2,3,
\end{array}\right.
$$

we conclude

$$
k_{x, \ell_{1}, \delta}+k_{x, \ell_{2}, \delta}+k_{x, \ell_{3}, \delta}-k_{x, \ell, \delta} \neq 0 .
$$

As a result, monomials of type " $C_{\ell_{1}} C_{\ell_{2}} C_{\ell_{3}}$ " are in the range of $\mathcal{D}-\mathrm{i} k_{x, \ell, \delta}$.

- Monomials of types " $C_{\ell_{1}} \overline{C_{-\ell_{2}} C_{-\ell_{3}}}$ " and " $\overline{C_{-\ell_{1}} C_{-\ell_{2}} C_{-\ell_{3}}}$ " are also in the range of $\mathcal{D}-\mathrm{i} k_{x, \ell}$. The proof is similar to the above case, essentially due to the fact that

$$
k_{x, \ell_{1}, \delta}-k_{x, \ell_{2}, \delta}-k_{x, \ell_{3}, \delta}-k_{x, \ell, \delta} \neq 0, \quad-k_{x, \ell_{1}, \delta}-k_{x, \ell_{2}, \delta}-k_{x, \ell_{3}, \delta}-k_{x, \ell, \delta} \neq 0 .
$$

- Monomials of type " $C_{\ell_{1}} C_{\ell_{2}} \overline{C_{-\ell_{3}}}$ " are not in the range of $\mathcal{D}-\mathrm{i} k_{x, \ell, \delta}$ precisely when

$$
\left\{\begin{array} { l } 
{ \ell _ { 1 } = \ell } \\
{ \ell _ { 2 } = - \ell _ { 3 } }
\end{array} \quad \text { or } \quad \left\{\begin{array}{l}
\ell_{1}=-\ell_{3} \\
\ell_{2}=\ell
\end{array}\right.\right.
$$

The proof again is similar to the first case but now relies on Lemma 2.5, which implies

$$
\left\{\begin{array} { l } 
{ \ell _ { 1 } + \ell _ { 2 } + \ell _ { 3 } - \ell = 0 } \\
{ k _ { x , \ell _ { 1 } , \delta } + k _ { x , \ell _ { 2 } , \delta } - k _ { x , \ell _ { 3 } , \delta } - k _ { x , \ell , \delta } = 0 }
\end{array} \quad \text { iff } \left\{\begin{array} { l } 
{ \ell _ { 1 } = \ell } \\
{ \ell _ { 2 } = - \ell _ { 3 } }
\end{array} \quad \text { or } \quad \left\{\begin{array}{l}
\ell_{1}=-\ell_{3} \\
\ell_{2}=\ell .
\end{array}\right.\right.\right.
$$

Consequently, upon choosing

$$
\Psi_{\ell}=-\frac{3 \mathrm{i}}{4 k_{x, \ell, \delta}^{3}} C_{\ell}\left(-\left|C_{\ell}\right|^{2}+2 \sum_{\kappa \in I_{*}}\left|C_{\kappa}\right|^{2}\right)+\widetilde{\Psi_{\ell}}
$$

with $\widetilde{\Psi_{\ell}}$ any element of the range of $\mathcal{D}-\mathrm{i} k_{x, \ell, \delta}$, the polynomial $\Psi_{\ell}+F_{\ell}$ belongs to the range of $\mathcal{D}-\mathrm{i} k_{x, \ell, \delta}$, so that there exists $\Phi_{\ell}$ satisfying the first equality in (2.19). Substituting (2.20) into the second equation in (2.19) we find

$$
\left(\mathcal{D}-\mathrm{i} k_{x, \ell, \delta}\right) \widetilde{\Psi_{\ell}}=\frac{3 \mathrm{i}}{4 k_{x, \ell, \delta}^{3}}\left(\mathcal{D}-\mathrm{i} k_{x, \ell}\right)\left(C_{\ell}\left(-\left|C_{\ell}\right|^{2}+2 \sum_{\kappa \in I_{*}}\left|C_{\kappa}\right|^{2}\right)\right)+G_{\ell}-N_{\ell} .
$$


Taking

$$
N_{\ell}=\frac{3 \mathrm{i}}{4 k_{x, \ell, \delta}^{3}}\left(\mathcal{D}-\mathrm{i} k_{x, \ell}\right)\left(C_{\ell}\left(-\left|C_{\ell}\right|^{2}+2 \sum_{\kappa \in I_{*}}\left|C_{\kappa}\right|^{2}\right)\right)+\frac{3}{4 k_{x, \ell, \delta}^{2}} C_{\ell}\left(-\left|C_{\ell}\right|^{2}+2 \sum_{\kappa \in I_{*}}\left|C_{\kappa}\right|^{2}\right),
$$

we find the equation

$$
\left(\mathcal{D}-\mathrm{i} k_{x, \ell, \delta}\right) \widetilde{\Psi_{\ell}}=\frac{1}{4 k_{x, \ell, \delta}^{2}}\left(P\left(\left(C_{\ell}, \overline{C_{\ell}}\right)_{\kappa \in I_{*}}\right)-3 C_{\ell}\left(-\left|C_{\ell}\right|^{2}+2 \sum_{\kappa \in I_{*}}\left|C_{\kappa}\right|^{2}\right)\right),
$$

with right hand side belonging to the range of $\mathcal{D}-\mathrm{i} k_{x, \ell, \delta}$. Consequently, $\widetilde{\Psi_{\ell}}$ exists, and it is not difficult to see that it also belongs to the range of $\mathcal{D}-\mathrm{i} k_{x, \ell, \delta}$.

Normal form of the reduced system. We apply the change of variables to the reduced system (2.10) and obtain the normal form to leading order as follows,

$$
\begin{aligned}
C_{\ell}^{\prime}= & \mathrm{i} \sqrt{\frac{1}{2}\left(k_{x, \ell, \delta}^{2}+\sqrt{k_{x, \ell, \delta}^{4}-\mu}\right)} C_{\ell}+D_{\ell} \\
D_{\ell}^{\prime}= & -\frac{1}{2}\left(k_{x, \ell, \delta}^{2}-\sqrt{k_{x, \ell, \delta}^{4}-\mu}\right) C_{\ell}+\mathrm{i} \sqrt{\frac{1}{2}\left(k_{x, \ell, \delta}^{2}+\sqrt{k_{x, \ell, \delta}^{4}-\mu}\right)} D_{\ell} \\
& +\frac{3}{4 k_{x, \ell, \delta}^{2}} C_{\ell}\left(-\left|C_{\ell}\right|^{2}+2 \sum_{\kappa \in I_{*}}\left|C_{\kappa}\right|^{2}\right) \\
& +\frac{3 \mathrm{i}}{4 k_{x, \ell, \delta}^{3}} D_{\ell}\left(-\left|C_{\ell}\right|^{2}+2 \sum_{\kappa \in I_{*}}\left|C_{\kappa}\right|^{2}\right) \\
& +\frac{3 \mathrm{i}}{4 k_{x, \ell, \delta}^{3}} C_{\ell}\left(-\left(C_{\ell} \overline{D_{\ell}}+\overline{C_{\ell}} D_{\ell}\right)+2 \sum_{\kappa \in I_{*}}\left(C_{\kappa} \overline{D_{\kappa}}+\overline{C_{\kappa}} D_{\kappa}\right)\right)
\end{aligned}
$$

The higher order terms in this normal form are of order

$$
|\mu|\left(\sum_{\kappa \in I_{*}}\left(\left|C_{\kappa}\right|+\left|D_{\kappa}\right|\right)\right)^{3}+\left(\sum_{\kappa \in I_{*}}\left(\left|C_{\kappa}\right|+\left|D_{\kappa}\right|\right)\right)^{5} .
$$

\subsection{Existence of heteroclinic orbits}

We next pass to a corotating frame with respect to the normal form symmetry, at leading order,

$$
C_{\ell}(x)=\exp \left(\tilde{\mathrm{i}}_{\ell, \delta, \mu} x\right) \widetilde{C_{\ell}}, \quad D_{\ell}(x)=\exp \left(\mathrm{i} \tilde{k}_{\ell, \delta, \mu} x\right) \widetilde{D_{\ell}}, \quad \ell \in I_{*},
$$

where $\tilde{k}_{\ell, \mu, \delta}=\sqrt{\frac{1}{2}\left(k_{x, \ell, \delta}^{2}+\sqrt{k_{x, \ell, \delta}^{4}-\mu}\right)}$. We can now scale the equation, explicitly exhibiting leadingorder terms:

$$
\widehat{x}=|\mu|^{1 / 2} x, \quad \widetilde{C_{\ell}}=|\mu|^{1 / 2} \widehat{C_{\ell}}, \quad \widetilde{D_{\ell}}=|\mu| \widehat{D_{\ell}}, \quad \ell \in I_{*},
$$

yields the new system, for $\ell \in I_{*}$,

$$
\begin{aligned}
C_{\ell}^{\prime} & =D_{\ell}+\mathcal{O}\left(|\mu|^{1 / 2}\right) \\
D_{\ell}^{\prime} & =-\frac{1}{4 k_{x, \ell, \delta}^{2}} C_{\ell}\left(\operatorname{sign}(\mu)-3\left(-\left|C_{\ell}\right|^{2}+2 \sum_{\kappa \in I_{*}}\left|C_{\kappa}\right|^{2}\right)\right)+\mathcal{O}\left(|\mu|^{1 / 2}\right),
\end{aligned}
$$


in which we have dropped the hats. Taking $\mu>0$, we can rewrite this as a second-order equation, for $\ell \in I_{*}$,

$$
C_{\ell}^{\prime \prime}=-\frac{1}{4 k_{x, \ell, \delta}^{2}} C_{\ell}+\frac{3}{4 k_{x, \ell, \delta}^{2}} C_{\ell}\left(-\left|C_{\ell}\right|^{2}+2 \sum_{\kappa \in I_{*}}\left|C_{\kappa}\right|^{2}\right)+\mathcal{O}\left(\mu^{1 / 2}\right) .
$$

We will proceed and find grain boundaries to leading order as heteroclinic solutions to (2.23). Note that this equation is non-autonomous at higher order, with non-autonomous terms induced by the corotating frame to terms that are not in normal form. Our approach therfore is based on three steps. We first identify asymptotic solutions exactly. In fact, (2.23) possesses a heteroclinic orbit connecting equilibria at leading order. These equilibria continue to periodic orbits at all orders since they correspond to rotated roll solutions. We will make this precise in the next paragraph. We will then identify the heteroclinic orbits at leading order and study the linearization at those. In the last step, we carry out perturbation theory by decomposing perturbations into "exactly" known terms at infinity, namely some representative of the family of roll solutions, and an unknown exponentially localized perturbation. Persistence then will follow from the implicit function theorem.

Rotated rolls. The Swift-Hohenberg equation (1.1) possesses roll solutions

$$
u_{\mu, \kappa}(x)=\sqrt{4\left(\mu-\kappa^{2}\right) / 3} \cos (\sqrt{1+\kappa} x)+\mathcal{O}\left(\left|\mu-\kappa^{2}\right|^{3 / 2}\right),
$$

for small $\mu \in\left(0, \mu_{0}\right]$ and $\kappa^{2}<\mu$ (see for instance [9]). By isotropy of the equation, we obtain a family of rotated rolls,

$$
\begin{aligned}
u_{\mu, \delta, \varepsilon, \ell}(x, y)= & \frac{1}{\sqrt{3}} \mu^{\frac{1}{2}}\left(1-4 k_{x, \ell, \delta}^{2} \varepsilon^{2}\right)^{\frac{1}{2}}\left(\mathrm{e}^{\mathrm{i}\left(\tilde{k}_{\ell, \mu, \delta}+\varepsilon \mu^{1 / 2}\right) x} \mathrm{e}^{\mathrm{i}\left(k_{*}+\delta\right) \ell y}\right. \\
& \left.+\mathrm{e}^{-\mathrm{i}\left(\tilde{k}_{\ell, \mu, \delta}+\varepsilon \mu^{1 / 2}\right) x} \mathrm{e}^{-\mathrm{i}\left(k_{*}+\delta\right) \ell y}\right)+\mathcal{O}\left(\mu^{3 / 2}+\varepsilon \mu\right),
\end{aligned}
$$

where we set

$$
\kappa=\left(\tilde{k}_{\ell, \mu, \delta}+\varepsilon \mu^{1 / 2}\right)^{2}-k_{x, \ell, \delta}^{2}, \quad \ell_{x}=\frac{\tilde{k}_{\ell, \mu, \delta}+\varepsilon \mu^{1 / 2}}{\sqrt{1+\kappa}}, \quad \ell_{y}=\frac{\left(k_{*}+\delta\right) \ell}{\sqrt{1+\kappa}}, \quad \ell \in I_{*},
$$

so that $\ell_{x}^{2}+\ell_{y}^{2}=1$.

These roll solutions correspond to solutions on the center manifold, which one readily finds in the form

$$
\mathbf{P}_{\mu, \delta, \varepsilon, \ell}(x)=\left(0, \ldots, \frac{1}{\sqrt{3}}\left(1-4 k_{x, \ell, \delta}^{2} \varepsilon^{2}\right)^{1 / 2} \mathrm{e}^{\mathrm{i} \varepsilon x}, \ldots, 0\right)+\mathcal{O}\left(\mu^{1 / 2}\right) .
$$

Here, the nonzero term is at the $\ell$-th position in a $2 \ell_{*}+1$-dimensional vector with position ordering $-\ell_{*}, \ldots, \ell_{*}$ from left to right. Notice that these periodic orbits are not reversible. In particular, the reversibility symmetry $\mathcal{R}$ generates a second family of periodic orbits

$$
\mathbf{Q}_{\mu, \delta, \varepsilon, \ell}(x)=\left(\mathcal{R} \mathbf{P}_{\mu, \delta, \varepsilon, \ell}\right)(-x)=\left(0, \ldots, \frac{1}{\sqrt{3}}\left(1-4 k_{x, \ell, \delta}^{2} \varepsilon^{2}\right)^{1 / 2} \mathrm{e}^{\mathrm{i} \varepsilon x}, \ldots, 0\right)+O\left(\mu^{1 / 2}\right),
$$

which corresponds to the reflected rolls $u_{\mu, \delta, \varepsilon, \ell}(-x, y)$ and the nonzero term is now at the $(-\ell)$-th position. 
Heteroclinic orbit of the leading order system. We set $\mu=\delta=0$ in (2.23). A priori, one can look for a large variety of heteroclinic solutions in this high-dimensional system of ODEs. Note that setting any of the modes $C_{\ell}=0$ provides us with invariant subspaces, so that we could in principal choose any two modes, $C_{\ell_{1}}$ and $C_{\ell_{2}}$, set $C_{\ell} \equiv 0$ for $\ell \notin\left\{\ell_{1}, \ell_{2}\right\}$, and attempt to find heteroclinic orbits connecting $\mathbf{P}_{\mu, \delta, \varepsilon, \ell_{1}}$ and $\mathbf{Q}_{\mu, \delta, \varepsilon, \ell_{2}}$. We focus on $\ell_{1}=-\ell_{2}=1$, here, which gives the simplest possible grain boundaries. In fact, requiring reflection symmetry imposes $\ell_{1}=-\ell_{2}$. Grain boundaries with $\ell_{1}>1$ correspond to patterns where multiple rolls fit inside the fixed-width strip in the $y$-direction, and can be found by changing $k_{*} \mapsto \ell_{1} k_{*}$ and subsequently considering $\ell_{1}=1$. From now on, we therefore just consider $\mathbf{P}_{\mu, \delta, \varepsilon, 1}$ and $\mathbf{Q}_{\mu, \delta, \varepsilon, 1}$.

We can then set $C_{\ell}=0$ for $|\ell| \neq 1$ and find

$$
\begin{aligned}
C_{1}^{\prime \prime} & =-\frac{1}{4 k_{x, 1}^{2}} C_{1}+\frac{3}{4 k_{x, 1}^{2}} C_{1}\left(\left|C_{1}\right|^{2}+2\left|C_{-1}\right|^{2}\right) \\
C_{-1}^{\prime \prime} & =-\frac{1}{4 k_{x, 1}^{2}} C_{-1}+\frac{3}{4 k_{x, 1}^{2}} C_{-1}\left(2\left|C_{1}\right|^{2}+\left|C_{-1}\right|^{2}\right) .
\end{aligned}
$$

According to [11] this system possesses a real, reversible heteroclinic orbit $\left(C_{+}^{*}, C_{-}^{*}\right)$ with the following properties:

(i) $\lim _{x \rightarrow \infty}\left(C_{+}^{*}(x), C_{-}^{*}(x)\right)=(1 / \sqrt{3}, 0)$ and $\lim _{x \rightarrow-\infty}\left(C_{+}^{*}(x), C_{-}^{*}(x)\right)=(0,1 / \sqrt{3})$;

(ii) $C_{+}^{*}(x) \geqslant 0$ and $C_{-}^{*}(x) \geqslant 0$, for all $x \in \mathbb{R}$;

(iii) $C_{+}^{*}(x)=C_{-}^{*}(-x)$, for all $x \in \mathbb{R}$;

(iv) $C_{+}^{*}(x)=C_{-}^{*}(x)$ if and only if $x=0$;

(v) $C_{+}^{* 2}(x)+C_{-}^{* 2}(x) \leqslant 1 / 3$ and $C_{+}^{*}(x)+C_{-}^{*}(x) \geqslant 1 / \sqrt{3}$, for all $x \in \mathbb{R}$.

Next, we study the linearization at this heteroclinic, which is

$$
\mathcal{L}_{*}\left(\begin{array}{c}
C_{-\ell_{*}} \\
\ldots \\
C_{-2} \\
C_{-1} \\
C_{0} \\
C_{1} \\
C_{2} \\
\ldots \\
C_{\ell_{*}}
\end{array}\right)=\left(\begin{array}{c}
C_{-\ell_{*}}^{\prime \prime}+\frac{1}{4 k_{x,-\ell_{*}}^{2}} C_{-\ell_{*}}-\frac{3}{2 k_{x,-\ell_{*}}^{2}}\left(C_{+}^{* 2}+C_{-}^{* 2}\right) C_{-\ell_{*}} \\
C_{-2}^{\prime \prime}+\frac{1}{4 k_{x,-2}^{2}} C_{-2}-\frac{3}{2 k_{x,-2}^{2}}\left(C_{+}^{* 2}+C_{-}^{* 2}\right) C_{-2} \\
C_{-1}^{\prime \prime}+\frac{1}{4 k_{x, 1}^{2}} C_{-1}-\frac{3}{4 k_{x, 1}^{2}}\left(2\left(C_{+}^{* 2}+C_{-}^{* 2}\right) C_{-1}+C_{-}^{* 2} \overline{C_{-1}}+2 C_{+}^{*} C_{-}^{*}\left(C_{1}+\overline{C_{1}}\right)\right) \\
C_{0}^{\prime \prime}+\frac{1}{4 k_{x, 0}^{2}} C_{\ell}-\frac{3}{2 k_{x, 0}^{2}}\left(C_{+}^{* 2}+C_{-}^{* 2}\right) C_{0} \\
C_{1}^{\prime \prime}+\frac{1}{4 k_{x, 1}^{2}} C_{1}-\frac{3}{4 k_{x, 1}^{2}}\left(2\left(C_{+}^{* 2}+C_{-}^{* 2}\right) C_{1}+C_{+}^{* 2} \overline{C_{1}}+2 C_{+}^{*} C_{-}^{*}\left(C_{-1}+\overline{C_{-1}}\right)\right) \\
C_{2}^{\prime \prime}+\frac{1}{4 k_{x, 2}^{2}} C_{2}-\frac{3}{2 k_{x, 2}^{2}}\left(C_{+}^{* 2}+C_{-}^{* 2}\right) C_{2} \\
\ldots \\
C_{\ell_{*}}^{\prime \prime}+\frac{1}{4 k_{x, \ell_{*}}^{2}} C_{\ell_{*}}-\frac{3}{2 k_{x, \ell_{*}}^{2}}\left(C_{+}^{* 2}+C_{-}^{* 2}\right) C_{\ell_{*}}
\end{array}\right) .
$$

We will see that $\mathcal{L}_{*}: \mathcal{Y}_{\eta}^{r} \rightarrow \mathcal{X}_{\eta}^{r}$ is Fredholm, where

$$
\begin{gathered}
\mathcal{X}_{\eta}^{r}=\left\{\left(C_{\ell}, \overline{C_{\ell}}\right)_{\ell \in I_{*}} \in \mathcal{X}_{\eta} ; C_{\ell}(x)=\overline{C_{-\ell}}(-x), x \in \mathbb{R}, \ell \in I_{*}\right\}, \\
\mathcal{X}_{\eta}=\left\{\left(C_{\ell}, \overline{C_{\ell}}\right)_{\ell \in I_{*}} \in\left(L_{\eta}^{2}\right)^{4 \ell+2}\right\}, \quad L_{\eta}^{2}=\left\{f: \mathbb{R} \rightarrow \mathbb{C} ; \int_{\mathbb{R}} \mathrm{e}^{2 \eta|x|}|f(x)|^{2}<\infty\right\},
\end{gathered}
$$




$$
\mathcal{Y}_{\eta}=\left\{\left(C_{\ell}, \overline{C_{\ell}}\right)_{\ell \in I_{*}} \in\left(H_{\eta}^{2}\right)^{4 \ell+2}\right\}, \quad H_{\eta}^{2}=\left\{f: \mathbb{R} \rightarrow \mathbb{C} ; f, f^{\prime}, f^{\prime \prime} \in L_{\eta}^{2}\right\},
$$

and $\mathcal{Y}_{\eta}^{r}=\mathcal{X}_{\eta}^{r} \cap \mathcal{Y}_{\eta}$.

Lemma 2.7 Assume $\eta>0$ is sufficiently small. Then the operator $\mathcal{L}_{*}: \mathcal{Y}_{\eta}^{r} \rightarrow \mathcal{X}_{\eta}^{r}$ is Fredholm with trivial kernel and one-dimensional co-kernel, spanned by

$$
\left(0, \ldots, 0,-\mathrm{i} C_{-}^{*}, 0, \mathrm{i} C_{+}^{*}, 0, \ldots, 0 ; 0, \ldots, 0, \mathrm{i} C_{-}^{*}, 0,-\mathrm{i} C_{+}^{*}, 0, \ldots, 0\right) .
$$

Proof. Since $C_{ \pm}^{*}$ is real, the linearization is diagonal after separating real and imaginary parts, $C_{\kappa}=U_{\kappa}+\mathrm{i} V_{\kappa}, \quad \kappa \in I_{*}$, with diagonal entries

$$
\begin{aligned}
\mathcal{M}_{\ell}\left(\begin{array}{c}
U_{\ell} \\
V_{\ell}
\end{array}\right) & =\left(\begin{array}{c}
U_{\ell}^{\prime \prime}+\frac{1}{4 k_{x, \ell}^{2}} U_{\ell}-\frac{3}{2 k_{x, \ell}^{2}}\left(C_{+}^{* 2}+C_{-}^{* 2}\right) U_{\ell} \\
V_{\ell}^{\prime \prime}+\frac{1}{4 k_{x, \ell}^{2}} V_{\ell}-\frac{3}{2 k_{x, \ell}^{2}}\left(C_{+}^{* 2}+C_{-}^{* 2}\right) V_{\ell}
\end{array}\right), \quad \text { for all } \ell \in I_{*} \backslash\{ \pm 1\} \\
\mathcal{M}_{r}\left(\begin{array}{c}
U_{1} \\
U_{-1}
\end{array}\right)= & \left(\begin{array}{c}
U_{1}^{\prime \prime}+\frac{1}{4 k_{x, 1}^{2}} U_{1}-\frac{3}{4 k_{x, 1}^{2}}\left(\left(3 C_{+}^{* 2}+2 C_{-}^{* 2}\right) U_{1}+4 C_{+}^{*} C_{-}^{*} U_{-1}\right) \\
U_{-1}^{\prime \prime}+\frac{1}{4 k_{x, 1}^{2}} U_{-1}-\frac{3}{4 k_{x, 1}^{2}}\left(\left(2 C_{+}^{* 2}+3 C_{-}^{* 2}\right) U_{-1}+4 C_{+}^{*} C_{-}^{*} U_{1}\right)
\end{array}\right), \\
\mathcal{M}_{i}\left(\begin{array}{c}
V_{1} \\
V_{-1}
\end{array}\right)= & \left(\begin{array}{c}
V_{1}^{\prime \prime}+\frac{1}{4 k_{x, 1}^{2}} V_{1}-\frac{3}{4 k_{x, 1}^{2}}\left(C_{+}^{* 2}+2 C_{-}^{* 2}\right) V_{1} \\
V_{-1}^{\prime \prime}+\frac{1}{4 k_{x, 1}^{2}} V_{-1}-\frac{3}{4 k_{x, 1}^{2}}\left(2 C_{+}^{* 2}+C_{-}^{* 2}\right) V_{-1}
\end{array}\right) .
\end{aligned}
$$

We can now follow [6] to conclude that all $\mathcal{M}_{\ell}$ are invertible, $\mathcal{M}_{i}$ is invertible, and $\mathcal{M}_{r}$ is Fredholm of index -1 . In fact, $\mathcal{M}_{r, i}$ coincide with the operators considered there, and we therefore also get an explicit description of the cokernel. This proves the lemma.

Persistence of the heteroclinic orbit. We are now ready to prove our main persistence result.

Theorem 2 Assume that $1 / k_{*} \notin \mathbb{Z}$. Then for all $\mu>0$ small and small angle variations $\delta$, there exists a smooth wavenumber correction $\varepsilon=\varepsilon(\sqrt{\mu}, \delta), \varepsilon(0,0)=0$, such that the system (2.23) possesses a heteroclinic orbit $\mathbf{C}_{\mu, \delta}$ connecting the periodic orbit $\mathbf{P}_{\mu, \delta, \varepsilon, 1}$ to $\mathbf{Q}_{\mu, \delta, \varepsilon, 1}$.

Proof. We outline the proof which essentially follows the strategy in [5,6]. We substitute into (2.23) together with its conjugate the ansatz

$$
\mathbf{C}(x)=\mathrm{e}^{\mathrm{i} \varepsilon x} \mathbf{C}^{*}(x)+\chi(x) \widetilde{\mathbf{P}}_{\mu, \delta, \varepsilon, 1}(x)+\left(\mathcal{R}\left(\chi \widetilde{\mathbf{P}}_{\mu, \delta, \varepsilon, 1}\right)\right)(-x)+\mathbf{V}(x),
$$

in which heteroclinic orbit and correction to the periodic orbit are given as

$$
\mathbf{C}^{*}=\left(0, \ldots, 0, C_{-}^{*}, 0, C_{+}^{*}, 0, \ldots, 0\right), \quad \widetilde{\mathbf{P}}_{\mu, \delta, \varepsilon, 1}=\mathbf{P}_{\mu, \delta, \varepsilon, 1}-\left(0, \ldots, 0,0,0, \frac{1}{\sqrt{3}}, 0, \ldots, 0\right) \mathrm{e}^{\mathrm{i} \varepsilon x},
$$

$\chi: \mathbb{R} \rightarrow[0,1]$ is a smooth cut-off function with

$$
\chi(x)=1, \text { if } x \geqslant M, \quad \chi(x)=0, \text { if } x \leqslant m, \quad \chi(x)+\chi(-x) \equiv 1,
$$

for some positive constants $m<M$, and $(\mathbf{V}, \overline{\mathbf{V}}) \in \mathcal{Y}_{\eta}^{r}$. The substitution then leads to a nonlinear equation of the form

$$
\mathcal{T}\left(\mathbf{V}, \overline{\mathbf{V}}, \varepsilon, \mu^{1 / 2}, \delta\right)=0
$$


In fact, $\mathcal{T}$ is smooth on the weighted spaces $\mathcal{Y}_{\eta}^{r}$ (here we use the particular form of the ansatz) and we have

$$
\mathcal{T}(0,0,0,0,0)=\mathcal{F}\left(\mathbf{C}^{*}, \overline{\mathbf{C}^{*}}, 0,0\right)=0, \quad D_{\mathbf{V}, \overline{\mathbf{V}}} \mathcal{T}(0,0,0,0,0)=\mathcal{L}_{*}
$$

and

$$
D_{\varepsilon} \mathcal{T}(0,0,0,0,0)=\mathcal{L}_{*}\left(\begin{array}{c}
i x \mathbf{C}^{*} \\
-i x \mathbf{C}^{*}
\end{array}\right)=\left(\begin{array}{c}
2 i \mathbf{C}^{* \prime} \\
-2 i \mathbf{C}^{* \prime}
\end{array}\right) .
$$

Using the explicit form of the co-kernel, we find that the linearization with respect to $\mathbf{V}, \overline{\mathbf{V}}$ and $\varepsilon$, jointly, is invertible, so that we can use the implicit function theorem to conclude persistence as stated in the theorem.

The result in Theorem 1 is an immediate consequence of Theorem 2. Since $\varepsilon=\mathcal{O}\left(\mu^{1 / 2}+|\delta|\right)$, we find that the angle of the selected grain boundary is $\alpha+\mathcal{O}(\mu+|\delta|)$, and that the wavenumber of the asymptotic rolls is $k_{x}=k_{x, 1}+\mathcal{O}\left(\mu+\mu^{1 / 2}|\delta|\right)$. As a result, to leading order in $\mu$ and $\delta$, the constant wavenumber $k$ has the expansion $k=\sqrt{k_{x}^{2}+\left(k_{*}+\delta\right)^{2}}=1+\mathcal{O}\left(\mu+\mu^{1 / 2}|\delta|\right)$, which, combined with the compactness of the interval $\left[\epsilon_{\varphi}, \pi-\epsilon_{\varphi}\right]$ for fixed $\epsilon_{\varphi} \in(0, \pi)$, shows that Theorem 1 is true.

\section{Grain boundaries for resonant angles $1 / k_{*} \in \mathbb{Z}$}

In this section, we treat the resonant case; that is, the wavenumber $k=k_{*}$ satisfies the condition that

$$
\frac{1}{k_{*}} \in \mathbb{Z}^{+} \backslash\{1\}
$$

Recall that this condition guarantees that rolls with angles $\varphi=0, \pm \arcsin \left(k_{*}\right), \ldots, \pm \arcsin \left(1-k_{*}\right), \pm \frac{\pi}{2}$ are compatible with the periodic boundary conditions in $y$. The basic idea follows the resonant case with some major differences that we will emphasize throughout, sometimes relegating the reader to Section 2 for aspects that are similar to the non-resonant case.

We start with the general center-manifold reduction and linear analysis in Section 3.1, calculate the normal form in Section 3.2, and prove persistence of heteroclinics in Section 3.3.

\subsection{Center manifold reduction}

Central space. We first recall the system (2.2)

$$
\frac{d U}{d x}=\mathcal{A}_{*} U+\mathcal{B}(\mu, \delta) U+\mathcal{F}(U)
$$

Taking into account the restriction (3.1) we have

$$
\sigma\left(\mathcal{A}_{*}\right) \cap \mathrm{i} \mathbb{R}=\left\{ \pm \mathrm{i} k_{\ell, x} \mid \ell=0, \pm 1, \ldots \pm \ell_{*}\right\}, \quad k_{x, \ell}=\sqrt{1-\left(k_{*} \ell\right)^{2}} \text { and } \ell_{*}=\frac{1}{k_{*}} .
$$

For simplicity, we denote $\widetilde{I}_{*}=\left\{0, \pm 1, \ldots \pm\left(\ell_{*}-1\right)\right\}$ and $J_{*}=\left\{ \pm \ell_{*}\right\}$. For every $\ell \in \widetilde{I}_{*}$, we define the generalized eigenspaces exactly as those in the nonresonant case. 
For $\jmath \in J$, the eigenvalue 0 is geometrically double and algebraically octuple. The generalized eigenspace associated with 0 is spanned by

$E_{ \pm \ell_{*}}(y)=\left(\begin{array}{l}1 \\ 0 \\ 0 \\ 0\end{array}\right) \mathrm{e}^{ \pm \mathrm{i} \ell_{*} y}, \quad F_{ \pm \ell_{*}, 1}(y)=\left(\begin{array}{l}0 \\ 1 \\ 0 \\ 0\end{array}\right) \mathrm{e}^{ \pm \mathrm{i} \ell_{*} y}, \quad F_{ \pm \ell_{*}, 2}(y)=\left(\begin{array}{l}0 \\ 0 \\ 1 \\ 0\end{array}\right) \mathrm{e}^{ \pm \mathrm{i} \ell_{*} y}, \quad F_{ \pm \ell_{*}, 3}(y)=\left(\begin{array}{l}0 \\ 0 \\ 0 \\ 1\end{array}\right) \mathrm{e}^{ \pm \mathrm{i} \ell_{*} y}$

which satisfy

$$
\mathcal{A}_{*} E_{ \pm \ell_{*}}=0, \quad \mathcal{A}_{*} F_{ \pm \ell_{*}, 1}=E_{ \pm \ell_{*}}, \quad \mathcal{A}_{*} F_{ \pm \ell_{*}, 2}=F_{ \pm \ell_{*}, 1}, \quad \mathcal{A}_{*} F_{ \pm \ell_{*}, 3}=F_{ \pm \ell_{*}, 2}
$$

The central space $\mathcal{X}_{c}$ of the operator $\mathcal{A}_{*}$ is $4\left(2 \ell_{*}+1\right)$-dimensional and spanned by the vectors

$$
\left\{E_{\ell}, F_{\ell}, \overline{E_{\ell}}, \overline{F_{\ell}} \mid \ell \in \widetilde{I}_{*}\right\} \bigcup\left\{E_{ \pm \ell_{*}}, F_{ \pm \ell_{*}, j} \mid j=1,2,3\right\}
$$

Moreover, the spectral projection $\mathcal{P}_{c}: \mathcal{X} \rightarrow \mathcal{X}_{c}$ is given by

$$
\begin{aligned}
\mathcal{P}_{c} U= & \sum_{\ell \in \widetilde{I}_{*}}\left(\left\langle U, E_{\ell}^{\mathrm{ad}}\right\rangle E_{\ell}+\left\langle U, F_{\ell}^{\mathrm{ad}}\right\rangle F_{\ell}+\left\langle U, \overline{E_{\ell}^{\mathrm{ad}}}\right\rangle \overline{E_{\ell}}+\left\langle U, \overline{F_{\ell}^{\mathrm{ad}}}\right\rangle \overline{F_{\ell}}\right)+ \\
& \frac{1}{2 \pi} \sum_{\jmath= \pm \ell_{*}}\left(\left\langle U, E_{\jmath}\right\rangle E_{\jmath}+\left\langle U, F_{\jmath, 1}\right\rangle F_{\jmath, 1}+\left\langle U, F_{\jmath, 2}\right\rangle F_{\jmath, 2}+\left\langle U, F_{\jmath, 3}\right\rangle F_{\jmath, 3}\right),
\end{aligned}
$$

where $\left\{E_{\ell}^{\mathrm{ad}}, F_{\ell}^{\mathrm{ad}}\right\}_{\ell \in \widetilde{I}_{*}}$ is defined exactly as those in the nonresonant case.

Reduction to a center manifold and reduced system. We reduce to a center manifold and expand the reduced system. A direct computation confirms that we have the same Taylor expansion as in the previous case

$$
\frac{d U_{c}}{d x}=\mathcal{A}_{*} U_{c}+\mathcal{P}_{c}\left(\mathcal{B}(\mu, \delta) U_{c}+\mathcal{F}\left(U_{c}\right)\right)+O\left(|\mu|\left\|U_{c}\right\|^{3}+\left\|U_{c}\right\|^{4}\right) .
$$

Next, using the basis of $\mathcal{X}_{c}$ constructed above we set

$$
\begin{aligned}
U_{c}(x)= & \sum_{\ell \in \widetilde{I}_{*}}\left(A_{\ell}(x) E_{\ell}+B_{\ell}(x) F_{\ell}+\overline{A_{\ell}}(x) \overline{E_{\ell}}+\overline{B_{\ell}}(x) \overline{F_{\ell}}\right)+ \\
& \sum_{\jmath= \pm \ell_{*}}\left(A_{\jmath}(x) E_{\jmath}+B_{\jmath, 1} F_{\jmath, 1}+B_{\jmath, 2} F_{\jmath, 2}+B_{\jmath, 3} F_{\jmath, 3}\right),
\end{aligned}
$$

where $A_{\ell}, B_{\ell}, A_{ \pm \ell_{*}}, B_{ \pm \ell_{*}, 1}, B_{ \pm \ell_{*}, 2}, B_{ \pm \ell_{*}, 3}, \ell \in \widetilde{I}_{*}$, are complex-valued functions and $A_{-\ell_{*}}=\overline{A_{\ell_{*}}}, B_{-\ell_{*}, j}=$ $\overline{B_{\ell_{*}, j}}, j=1,2,3$. A straightforward but lengthy calculation gives the leading order terms in the reduced system

$$
\frac{d U_{c}}{d x}=\mathcal{A}_{*} U_{c}+\mathcal{P}_{c}\left(\mathcal{B}(\mu, \delta) U_{c}+\mathcal{F}\left(U_{c}\right)\right),
$$


expressed in the basis $\left\{A_{\ell}, B_{\ell}, A_{\ell_{*}}, B_{\ell_{*}, 1}, B_{\ell_{*}, 2}, B_{\ell_{*}, 3} \mid \ell \in \widetilde{I}_{*}\right\}$ of the center eigenspace,

$$
\begin{aligned}
A_{\ell}^{\prime} & =\mathrm{i} k_{x, \ell} A_{\ell}+B_{\ell}-\mathrm{i} \frac{\mu+2 k_{x, \ell}^{2}\left(k_{x, \ell}^{2}-k_{x, \ell, \delta}^{2}\right)}{4 k_{x, \ell}^{3}} a_{\ell}+\frac{k_{x, \ell}^{2}-k_{x, \ell, \delta}^{2}}{2 k_{x, \ell}^{2}} b_{\ell}+\frac{\mathrm{i}}{4 k_{x, \ell}^{3}} P_{\ell} \\
B_{\ell}^{\prime} & =\mathrm{i} k_{x, \ell} B_{\ell}-\frac{1}{4 k_{x, \ell}^{2}} \mu a_{\ell}-\mathrm{i} \frac{k_{x, \ell}^{2}-k_{x, \ell, \delta}^{2}}{2 k_{x, \ell}} b_{\ell}+\frac{1}{4 k_{x, \ell}^{2}} P_{\ell} \\
A_{\ell_{*}}^{\prime} & =B_{\ell_{*}, 1} \\
B_{\ell_{*}, 1}^{\prime} & =-k_{x, \ell_{*}, \delta}^{2} A_{\ell_{*}}+B_{\ell_{*}, 2} \\
B_{\ell_{*}, 2}^{\prime} & =B_{\ell_{*}, 3} \\
B_{\ell_{*}, 2}^{\prime} & =\mu A_{\ell_{*}}-k_{x, \ell_{*}, \delta}^{2} B_{\ell_{*}, 2}-P_{\ell_{*}}
\end{aligned}
$$

in which

$$
k_{x, \ell_{*}, \delta}^{2}=1-\left(k_{*}+\delta\right)^{2} \ell_{*}^{2}, \quad P_{\ell}\left(\left(A_{\ell}, \overline{A_{\ell}}\right)_{\ell \in I_{*}}\right)=\sum_{\substack{\ell_{1}+\ell_{2}+\ell_{3}=\ell, \ell_{1}, \ell_{2}, \ell_{3} \in I_{*}}} a_{\ell_{1}} a_{\ell_{2}} a_{\ell_{3}}, \quad \text { for all } \ell \in I_{*},
$$

where

$$
k_{x, \ell_{*}, \delta}=\left\{\begin{array}{ll}
\sqrt{1-\left(k_{*}+\delta\right)^{2} \ell_{*}^{2}}, & \delta \leqslant 0 \\
-\mathrm{i} \sqrt{\left(k_{*}+\delta\right)^{2} \ell_{*}^{2}-1}, & \delta>0,
\end{array} \quad a_{\ell}= \begin{cases}A_{\ell}+\overline{A_{-\ell}}, & \ell \in \widetilde{I}_{*} \\
A_{\ell_{*}}, & \ell=\ell_{*} \\
A_{-\ell_{*}}, & \ell=-\ell_{*} .\end{cases}\right.
$$

Note that here $|\delta|$ is sufficiently small and $k_{x, \ell_{*}, \delta}$ is not smooth with respect to $\delta$ while $k_{x, \ell_{*}, \delta}^{2}$ is.

Symmetries. The three reflection symmetries in the Swift-Hohenberg equation

$$
y \mapsto-y, \quad x \mapsto-x, \quad u \mapsto-u .
$$

are inherited through the center manifold reduction, that is, the reduced system (3.3) inherits the induced symmetries. While the symmetries with respect to the $\left(A_{\ell}, B_{\ell}, \overline{A_{\ell}}, \overline{B_{\ell}}\right)_{\ell \in \widetilde{I}_{*}}$ are the same as those in the nonresonant case, we have to take care of the other part here as follows. The reflection $y \mapsto-y, u \mapsto-u$ and the invariance of the Swift-Hohenberg equation (1.3) under translations in $y$ imply symmetries in the reduced system (3.3) that

$$
\begin{aligned}
& \mathcal{S}_{1}\left(A_{\ell_{*}}, B_{\ell_{*}, 1}, B_{\ell_{*}, 2}, B_{\ell_{*}, 3}, A_{-\ell_{*}}, B_{-\ell_{*}, 1}, B_{-\ell_{*}, 2}, B_{-\ell_{*}, 3}\right)^{T} \\
&=\left(A_{-\ell_{*}}, B_{-\ell_{*}, 1}, B_{-\ell_{*}, 2}, B_{-\ell_{*}, 3}, A_{\ell_{*}}, B_{\ell_{*}, 1}, B_{\ell_{*}, 2}, B_{\ell_{*}, 3}\right)^{T}, \\
& \mathcal{S}_{2}\left(A_{\ell_{*}}, B_{\ell_{*}, 1}, B_{\ell_{*}, 2}, B_{\ell_{*}, 3}, A_{-\ell_{*}}, B_{-\ell_{*}, 1}, B_{-\ell_{*}, 2}, B_{-\ell_{*}, 3}\right)^{T} \\
&=-\left(A_{\ell_{*}}, B_{\ell_{*}, 1}, B_{\ell_{*}, 2}, B_{\ell_{*}, 3}, A_{-\ell_{*}}, B_{-\ell_{*}, 1}, B_{-\ell_{*}, 2}, B_{-\ell_{*}, 3}\right)^{T}, \\
& \mathcal{T}_{\phi}\left(A_{\ell_{*}}, B_{\ell_{*}, 1}, B_{\ell_{*}, 2}, B_{\ell_{*}, 3}, A_{-\ell_{*}}, B_{-\ell_{*}, 1}, B_{-\ell_{*}, 2}, B_{-\ell_{*}, 3}\right)^{T} \\
&=\left(\mathrm{e}^{i \ell_{*} \phi} A_{\ell_{*}}, \mathrm{e}^{i \ell_{*} \phi} B_{\ell_{*}, 1}, \mathrm{e}^{i \ell_{*} \phi} B_{\ell_{*}, 2}, \mathrm{e}^{i \ell_{*} \phi} B_{\ell_{*}, 3}, \mathrm{e}^{-i \ell_{*} \phi} A_{-\ell_{*}}, \mathrm{e}^{-i \ell_{*} \phi} B_{-\ell_{*}, 1}, \mathrm{e}^{-i \ell_{*} \phi} B_{-\ell_{*}, 2}, \mathrm{e}^{-i \ell_{*} \phi} B_{-\ell_{*}, 3}\right)^{T} .
\end{aligned}
$$

The reflection $x \mapsto-x$ implies that the vector field of the reduced system anticommutes with

$$
\begin{gathered}
\mathcal{R}\left(A_{\ell_{*}}, B_{\ell_{*}, 1}, B_{\ell_{*}, 2}, B_{\ell_{*}, 3}, A_{-\ell_{*}}, B_{-\ell_{*}, 1}, B_{-\ell_{*}, 2}, B_{-\ell_{*}, 3}\right)^{T} \\
=\left(A_{\ell_{*}},-B_{\ell_{*}, 1}, B_{\ell_{*}, 2},-B_{\ell_{*}, 3}, A_{-\ell_{*}},-B_{-\ell_{*}, 1}, B_{-\ell_{*}, 2},-B_{-\ell_{*}, 3}\right)^{T} .
\end{gathered}
$$


As a consequence, the higher order terms in the reduced system (3.3) are such that

$$
\frac{d U_{c}}{d x}=\mathcal{A}_{*} U_{c}+\mathcal{P}_{c}\left(\mathcal{B}(\mu, \delta) U_{c}+\mathcal{F}\left(U_{c}\right)\right)+\mathcal{O}\left(|\mu|\left\|U_{c}\right\|^{3}+\left\|U_{c}\right\|^{5}\right)
$$

\subsection{Normal form transformations}

Just as in Section 2.3, to arrive at a normal form for (3.5), we sequentially simplifying the linear terms and the cubic terms.

Linear versal transformation. Consider the linear part of the system (3.4), for $\ell \in \widetilde{I}_{*}$,

$$
\begin{aligned}
A_{\ell}^{\prime} & =\mathrm{i} k_{x, \ell} A_{\ell}+B_{\ell}-\mathrm{i} \frac{\mu+2 k_{x, \ell}^{2}\left(k_{x, \ell}^{2}-k_{x, \ell, \delta}^{2}\right)}{4 k_{x, \ell}^{3}} a_{\ell}+\frac{k_{x, \ell}^{2}-k_{x, \ell, \delta}^{2}}{2 k_{x, \ell}^{2}} b_{\ell} \\
B_{\ell}^{\prime} & =\mathrm{i} k_{x, \ell} B_{\ell}-\frac{1}{4 k_{x, \ell}^{2}} \mu a_{\ell}-\mathrm{i} \frac{k_{x, \ell}^{2}-k_{x, \ell, \delta}^{2}}{2 k_{x, \ell}} b_{\ell} \\
A_{\ell_{*}}^{\prime} & =B_{\ell_{*}, 1} \\
B_{\ell_{*}, 1}^{\prime} & =-k_{x, \ell_{*}, \delta}^{2} A_{\ell_{*}}+B_{\ell_{*}, 2} \\
B_{\ell_{*}, 2}^{\prime} & =B_{\ell_{*}, 3} \\
B_{\ell_{*}, 2}^{\prime} & =\mu A_{\ell_{*}}-k_{x, \ell_{*}, \delta}^{2} B_{\ell_{*}, 2}-P_{\ell_{*}}
\end{aligned}
$$

We derive the linear normal form for the non-resonant modes $\left(A_{\ell}, B_{\ell}, \overline{A_{\ell}}, \overline{B_{\ell}}\right)_{\ell \in \widetilde{I}_{*}}$ just like in the nonresonant case, Lemma 2.4. For $\left(A_{\jmath}, B_{\jmath, 1}, B_{\jmath, 2}, B_{\jmath, 3}\right)_{\jmath= \pm \ell_{*}}$, we have the following unfolding.

Lemma 3.1 There exist linear maps $L_{\ell_{*}}(\mu, \delta)$ such that, for sufficiently small $\mu$ and $\delta$, the linear change of variables

$$
\begin{gathered}
\left(A_{\ell_{*}}, B_{\ell_{*}, 1}, B_{\ell_{*}, 2}, B_{\ell_{*}, 3}, A_{-\ell_{*}}, B_{-\ell_{*}, 1}, B_{-\ell_{*}, 2}, B_{-\ell_{*}, 3}\right)^{T} \\
=L_{\ell_{*}}(\mu, \delta)\left(C_{\ell_{*}}, D_{\ell_{*}, 1}, C_{\ell_{*}, 2}, D_{\ell_{*}, 3}, C_{-\ell_{*}}, D_{-\ell_{*}, 1}, C_{-\ell_{*}, 2}, D_{-\ell_{*}, 3}\right)^{T}
\end{gathered}
$$

transforms the corresponding part of system (3.6) together with its complex conjugate into the normal form

$$
\begin{aligned}
C_{\jmath}^{\prime} & =D_{\jmath, 1} \\
D_{\jmath, 1}^{\prime} & =D_{\jmath, 2} \\
D_{\jmath, 2}^{\prime} & =D_{\jmath, 3} \\
D_{\jmath, 3}^{\prime} & =\left(\mu-k_{x, \ell_{*}, \delta}^{4}\right) C_{\jmath}-2 k_{x, \ell_{*}, \delta}^{2} D_{\jmath, 2}
\end{aligned}
$$


where $\jmath= \pm \ell_{*}$. Moreover, we can choose $L_{\ell}(\mu, \delta)$ as

$$
\left(\begin{array}{cccccccc}
1 & 0 & 0 & 0 & 0 & 0 & 0 & 0 \\
0 & 1 & 0 & 0 & 0 & 0 & 0 & 0 \\
k_{x, \ell_{*}, \delta}^{2} & 0 & 1 & 0 & 0 & 0 & 0 & 0 \\
0 & k_{x, \ell_{*}, \delta}^{2} & 0 & 1 & 0 & 0 & 0 & 0 \\
0 & 0 & 0 & 0 & 1 & 0 & 0 & 0 \\
0 & 0 & 0 & 0 & 0 & 1 & 0 & 0 \\
0 & 0 & 0 & 0 & k_{x, \ell_{*}, \delta}^{2} & 0 & 1 & 0 \\
0 & 0 & 0 & 0 & 0 & k_{x, \ell_{*}, \delta}^{2} & 0 & 1
\end{array}\right) .
$$

Proof. We proceed as in the proof of Lemma 3.2. The versal transformation we use here is just the Sylvester family (see [1] for details). To obtain $L_{\ell_{*}}(\mu, \delta)$ as claimed, just note the fact that $L_{\ell_{*}}(\mu, \delta)$ commutes with $\mathcal{S}_{1}, \mathcal{S}_{2}, \mathcal{R}$ and $\mathcal{T}_{\phi}$ for all $\phi \in \mathbb{R}$.

Based on Lemma 3.1, our reduced system $(3.4,3.5)$ has the following expression. For all $\ell \in \widetilde{I}_{*}$,

$$
\begin{aligned}
C_{\ell}^{\prime} & =\mathrm{i} \sqrt{\frac{1}{2}\left(k_{x, \ell, \delta}^{2}+\sqrt{k_{x, \ell, \delta}^{4}-\mu}\right)} C_{\ell}+D_{\ell}+\frac{\mathrm{i}}{4 k_{x, \ell, \delta}^{3}} P_{\ell} \\
D_{\ell}^{\prime} & =-\frac{1}{2}\left(k_{x, \ell, \delta}^{2}-\sqrt{k_{x, \ell, \delta}^{4}-\mu}\right) C_{\ell}+\mathrm{i} \sqrt{\frac{1}{2}\left(k_{x, \ell, \delta}^{2}+\sqrt{k_{x, \ell, \delta}^{4}-\mu}\right)} D_{\ell}+\frac{1}{4 k_{x, \ell, \delta}^{2}} P_{\ell} \\
C_{\ell_{*}}^{\prime} & =D_{\ell_{*}, 1} \\
D_{\ell_{*}, 1}^{\prime} & =D_{\ell_{*}, 2} \\
D_{\ell_{*}, 2}^{\prime} & =D_{\ell_{*}, 3} \\
D_{\ell_{*}, 3}^{\prime} & =\left(\mu-k_{x, \ell_{*}, \delta}^{4}\right) C_{\ell_{*}}-2 k_{x, \ell_{*}, \delta}^{2} D_{\ell_{*}, 2}-P_{\ell_{*}} .
\end{aligned}
$$

with higher order terms in the form

$$
\begin{gathered}
\mathcal{O}\left(|\mu|\left(\sum_{\kappa \in \widetilde{I}_{*}}\left|C_{\kappa}\right|+\left|D_{\kappa}\right|+\sum_{\zeta= \pm \ell_{*}}\left|C_{\zeta}\right|+\left|D_{\zeta, 1}\right|+\left|D_{\zeta, 2}\right|+\left|D_{\zeta, 3}\right|\right)^{3}\right. \\
\left.+\left(\sum_{\kappa \in \widetilde{I}_{*}}\left|C_{\kappa}\right|+\left|D_{\kappa}\right|+\sum_{\zeta= \pm \ell_{*}}\left|C_{\zeta}\right|+\left|D_{\zeta, 1}\right|+\left|D_{\zeta, 2}\right|+\left|D_{\zeta, 3}\right|\right)^{5}\right) .
\end{gathered}
$$

Cubic transformation. Taking $\mu=\delta=0$, we obtain the leading order of system (2.9),

$$
\begin{aligned}
A_{\ell}^{\prime} & =\mathrm{i} k_{x, \ell} A_{\ell}+B_{\ell}+\frac{\mathrm{i}}{4 k_{x, \ell}^{3}} P_{\ell} \\
B_{\ell}^{\prime} & =\mathrm{i} k_{x, \ell} B_{\ell}+\frac{1}{4 k_{x, \ell}^{2}} P_{\ell} \\
A_{\ell_{*}}^{\prime} & =B_{\ell_{*}, 1} \\
B_{\ell_{*}, 1}^{\prime} & =B_{\ell_{*}, 2} \\
B_{\ell_{*}, 2}^{\prime} & =B_{\ell_{*}, 3} \\
B_{\ell_{*}, 2}^{\prime} & =-P_{\ell_{*} .}
\end{aligned}
$$


Lemma 3.2 There exist homogeneous polynomials $\left\{\Phi_{\ell}, \Psi_{\ell}, \Phi_{\ell_{*}}, \Psi_{\ell_{*}, 1}, \Psi_{\ell_{*}, 2}, \Psi_{\ell_{*}, 3} \mid \ell \in \widetilde{I}_{*}\right\}$ of degree 3 in the complex variables $\left(C_{\kappa}, D_{\kappa}, \overline{C_{\kappa}}, \overline{D_{\kappa}}\right)_{\kappa \in I_{*}}$ and $\left(C_{\zeta}, D_{\zeta, 1}, D_{\zeta, 2}, D_{\zeta, 3}\right)_{\zeta= \pm \ell_{*}}$, such that the change of variables, for all $\ell \in \widetilde{I}_{*}$,

$$
\begin{aligned}
A_{\ell} & =C_{\ell}+\Phi_{\ell}\left(\left(C_{\kappa}, D_{\kappa}, \overline{C_{\kappa}}, \overline{D_{\kappa}}\right)_{\kappa \in \widetilde{I}_{*}},\left(C_{\zeta}, D_{\zeta, 1}, D_{\zeta, 2}, D_{\zeta, 3}\right)_{\zeta= \pm \ell_{*}}\right), \\
B_{\ell} & =D_{\ell}+\Psi_{\ell}\left(\left(C_{\kappa}, D_{\kappa}, \overline{C_{\kappa}}, \overline{D_{\kappa}}\right)_{\kappa \in \widetilde{I}_{*}},\left(C_{\zeta}, D_{\zeta, 1}, D_{\zeta, 2}, D_{\zeta, 3}\right)_{\zeta= \pm \ell_{*}}\right), \\
A_{\ell_{*}} & =C_{\ell_{*}}+\Phi_{\ell_{*}}\left(\left(C_{\kappa}, D_{\kappa}, \overline{C_{\kappa}}, \overline{D_{\kappa}}\right)_{\kappa \in \widetilde{I}_{*}},\left(C_{\zeta}, D_{\zeta, 1}, D_{\zeta, 2}, D_{\zeta, 3}\right)_{\zeta= \pm \ell_{*}}\right), \\
B_{\ell_{*}, 1} & =D_{\ell_{*}, 1}+\Psi_{\ell_{*}, 1}\left(\left(C_{\kappa}, D_{\kappa}, \overline{C_{\kappa}}, \overline{D_{\kappa}}\right)_{\kappa \in \widetilde{I}_{*}},\left(C_{\zeta}, D_{\zeta, 1}, D_{\zeta, 2}, D_{\zeta, 3}\right)_{\zeta= \pm \ell_{*}}\right), \\
B_{\ell_{*}, 2} & =D_{\ell_{*}, 2}+\Psi_{\ell_{*}, 2}\left(\left(C_{\kappa}, D_{\kappa}, \overline{C_{\kappa}}, \overline{D_{\kappa}}\right)_{\kappa \in \widetilde{I}_{*}},\left(C_{\zeta}, D_{\zeta, 1}, D_{\zeta, 2}, D_{\zeta, 3}\right)_{\zeta= \pm \ell_{*}}\right), \\
B_{\ell_{*}, 3} & =D_{\ell_{*}, 3}+\Psi_{\ell_{*}, 3}\left(\left(C_{\kappa}, D_{\kappa}, \overline{C_{\kappa}}, \overline{D_{\kappa}}\right)_{\kappa \in \widetilde{I}_{*}},\left(C_{\zeta}, D_{\zeta, 1}, D_{\zeta, 2}, D_{\zeta, 3}\right)_{\zeta= \pm \ell_{*}}\right),
\end{aligned}
$$

is well-defined in a neighborhood of the origin and transforms the system (2.16) into the normal form with $\ell \in \widetilde{I}_{*}$,

$$
\begin{aligned}
C_{\ell}^{\prime}= & \mathrm{i} k_{x, \ell} C_{\ell}+D_{\ell}+\mathcal{O}\left(\left(\sum_{\kappa \in \widetilde{I}_{*}}\left|C_{\kappa}\right|+\left|D_{\kappa}\right|+\sum_{\zeta= \pm \ell_{*}}\left|C_{\zeta}\right|+\left|D_{\zeta, 1}\right|+\left|D_{\zeta, 2}\right|+\left|D_{\zeta, 3}\right|\right)^{5}\right) \\
D_{\ell}^{\prime}= & \mathrm{i} k_{x, \ell} D_{\ell}+\frac{3}{4 k_{x, \ell}^{2}} C_{\ell}\left(\left|C_{\ell_{*}}\right|^{2}-\left|C_{\ell}\right|^{2}+2 \sum_{\kappa \in \widetilde{I}_{*}}\left|C_{\kappa}\right|^{2}\right)+\frac{3 \mathrm{i}}{4 k_{x, \ell}^{3}} D_{\ell}\left(\left|C_{\ell_{*}}\right|^{2}-\left|C_{\ell}\right|^{2}+2 \sum_{\kappa \in \widetilde{I}_{*}}\left|C_{\kappa}\right|^{2}\right) \\
& +\frac{3 \mathrm{i}}{4 k_{x, \ell}^{3}} C_{\ell}\left(\left(C_{\ell_{*}} \overline{D_{\ell_{*}, 1}}+\overline{C_{\ell_{*}}} D_{\ell_{*}, 1}\right)-\left(C_{\ell} \overline{D_{\ell}}+\overline{C_{\ell}} D_{\ell}\right)+2 \sum_{\kappa \in I_{*}}\left(C_{\kappa} \overline{D_{\kappa}}+\overline{C_{\kappa}} D_{\kappa}\right)\right) \\
& +\mathcal{O}\left(\left(\sum_{\kappa \in \widetilde{I}_{*}}\left|C_{\kappa}\right|+\left|D_{\kappa}\right|+\sum_{\zeta= \pm \ell_{*}}\left|C_{\zeta}\right|+\left|D_{\zeta, 1}\right|+\left|D_{\zeta, 2}\right|+\left|D_{\zeta, 3}\right|\right)^{5}\right) \\
C_{\ell_{*}}^{\prime}= & D_{\ell_{*}, 1} \\
D_{\ell_{*}, 1}^{\prime}= & D_{\ell_{*}, 2} \\
D_{\ell_{*}, 2}^{\prime}= & D_{\ell_{*}, 3} \\
D_{\ell_{*}, 3}^{\prime}= & -3 C_{\ell_{*}}\left(\left|C_{\ell_{*}}\right|^{2}+2 \sum_{\kappa \in \widetilde{I}_{*}}\left|C_{\kappa}\right|^{2}\right)+\mathcal{O}\left(\left(\sum_{\kappa \in \widetilde{I}_{*}}\left|C_{\kappa}\right|+\left|D_{\kappa}\right|+\sum_{\zeta= \pm \ell_{*}}\left|C_{\zeta}\right|+\left|D_{\zeta, 1}\right|+\left|D_{\zeta, 2}\right|+\left|D_{\zeta, 3}\right|\right)^{5}\right) .
\end{aligned}
$$

Proof. Denote the cubic terms in the right hand sides of the systems (3.11) and (3.13), respectively, by $\left(F_{\ell}, G_{\ell}\right)_{\ell \in \widetilde{I}_{*}}$ and $\left(0,0,0, G_{\ell_{*}, 3}\right),\left(0, N_{\ell}\right)_{\ell \in \widetilde{I}_{*}}$ and $\left(0,0,0, N_{\ell_{*}, 3}\right)$. Using the same argument as in the nonresonant case, we derive $\left(0, N_{\ell}\right)_{\ell \in \tilde{I}_{*}}$. The constant of $\left|C_{\ell_{*}}\right|^{2}$ is 1 instead of 2 due to the fact that $a_{ \pm \ell_{*}}=A_{ \pm \ell_{*}}$ instead of $a_{ \pm \ell_{*}}=A_{ \pm \ell_{*}}+\overline{A_{\mp \ell_{*}}}$. On the other hand, it is straightforward to see that

$$
\Phi_{\ell_{*}}=0, \quad \Psi_{\ell_{*}, 1}=0, \quad \Psi_{\ell_{*}, 2}=0 .
$$

Moreover, we have

$$
\mathcal{D} \Psi_{\ell_{*}, 3}=G_{\ell_{*}, 3}-N_{\ell_{*}, 3}
$$

where

$$
\begin{aligned}
\mathcal{D}= & \sum_{\ell \in I_{*}}\left(\left(\mathrm{i} k_{x, \ell} C_{\ell}+D_{\ell}\right) \frac{\partial}{\partial C_{\ell}}+\mathrm{i} k_{x, \ell} D_{\ell} \frac{\partial}{\partial D_{\ell}}+\left(-\mathrm{i} k_{x, \ell} \overline{C_{\ell}}+\overline{D_{\ell}}\right) \frac{\partial}{\partial \overline{C_{\ell}}}+\left(-\mathrm{i} k_{x, \ell} \overline{D_{\ell}}\right) \frac{\partial}{\partial \overline{D_{\ell}}}\right)+ \\
& \sum_{\jmath= \pm \ell_{*}}\left(D_{\jmath, 1} \frac{\partial}{\partial C_{\jmath}}+D_{\jmath, 2} \frac{\partial}{\partial D_{\jmath, 1}}+D_{\jmath, 2} \frac{\partial}{\partial D_{\jmath, 3}}\right) .
\end{aligned}
$$


Then it is not hard to see that

$$
N_{\ell_{*}, 3}=-3 C_{\ell_{*}}\left(\left|C_{\ell_{*}}\right|^{2}+2 \sum_{\kappa \in \widetilde{I}_{*}}\left|C_{\kappa}\right|^{2}\right) .
$$

Normal form of the reduced system. Applying the change of variables to the reduced system (3.5) gives the normal form to leading order, for $\ell \in \widetilde{I}_{*}$,

$$
\begin{aligned}
C_{\ell}^{\prime}= & \mathrm{i} \sqrt{\frac{1}{2}\left(k_{x, \ell, \delta}^{2}+\sqrt{k_{x, \ell, \delta}^{4}-\mu}\right)} C_{\ell}+D_{\ell} \\
D_{\ell}^{\prime}= & -\frac{1}{2}\left(k_{x, \ell, \delta}^{2}-\sqrt{k_{x, \ell, \delta}^{4}-\mu}\right) C_{\ell}+\mathrm{i} \sqrt{\frac{1}{2}\left(k_{x, \ell, \delta}^{2}+\sqrt{k_{x, \ell, \delta}^{4}-\mu}\right)} D_{\ell} \\
& +\frac{3}{4 k_{x, \ell}^{2}} C_{\ell}\left(-\left|C_{\ell}\right|^{2}+2 \sum_{\kappa \in I_{*}}\left|C_{\kappa}\right|^{2}\right) \\
& +\frac{3 \mathrm{i}}{4 k_{x, \ell}^{3}} D_{\ell}\left(-\left|C_{\ell}\right|^{2}+2 \sum_{\kappa \in I_{*}}\left|C_{\kappa}\right|^{2}\right) \\
& +\frac{3 \mathrm{i}}{4 k_{x, \ell}^{3}} C_{\ell}\left(-\left(C_{\ell} \overline{D_{\ell}}+\overline{C_{\ell}} D_{\ell}\right)+2 \sum_{\kappa \in I_{*}}\left(C_{\kappa} \overline{D_{\kappa}}+\overline{C_{\kappa}} D_{\kappa}\right)\right) \\
C_{\ell_{*}}^{\prime}= & D_{\ell_{*}, 1} \\
D_{\ell_{*}, 1}^{\prime}= & D_{\ell_{*}, 2} \\
D_{\ell_{*}, 2}^{\prime}= & D_{\ell_{*}, 3} \\
D_{\ell_{*}, 3}^{\prime}= & \left(\mu-k_{x, \ell_{*}, \delta}^{4}\right) C_{\ell_{*}}-2 k_{x, \ell_{*}, \delta}^{2} D_{\ell_{*}, 2}-3 C_{\ell_{*}}\left(\left|C_{\ell_{*}}\right|^{2}+2 \sum_{\kappa \in \widetilde{I}_{*}}\left|C_{\kappa}\right|^{2}\right) .
\end{aligned}
$$

The higher order terms in this normal form are of order

$$
\begin{gathered}
|\delta|\left(\sum_{\kappa \in \widetilde{I}_{*}}\left(\left|C_{\kappa}\right|+\left|D_{\kappa}\right|\right)+\sum_{\zeta= \pm \ell_{*}}\left(\left|C_{\zeta}\right|+\left|D_{\zeta, 1}\right|+\left|D_{\zeta, 2}\right|+\left|D_{\zeta, 3}\right|\right)\right)^{3}+ \\
|\mu|\left(\sum_{\kappa \in \widetilde{I}_{*}}\left(\left|C_{\kappa}\right|+\left|D_{\kappa}\right|\right)+\sum_{\zeta= \pm \ell_{*}}\left(\left|C_{\zeta}\right|+\left|D_{\zeta, 1}\right|+\left|D_{\zeta, 2}\right|+\left|D_{\zeta, 3}\right|\right)\right)^{3}+ \\
\quad\left(\sum_{\kappa \in \widetilde{I}_{*}}\left(\left|C_{\kappa}\right|+\left|D_{\kappa}\right|\right)+\sum_{\zeta= \pm \ell_{*}}\left(\left|C_{\zeta}\right|+\left|D_{\zeta, 1}\right|+\left|D_{\zeta, 2}\right|+\left|D_{\zeta, 3}\right|\right)\right)^{5} .
\end{gathered}
$$

Specifically, the higher order terms for $\left(C_{\ell_{*}}, D_{\ell_{*}, 1}, D_{\ell_{*}, 2}, D_{\ell_{*}, 3}\right)$ are of order

$$
\begin{gathered}
|\mu|\left(\sum_{\kappa \in \widetilde{I}_{*}}\left(\left|C_{\kappa}\right|+\left|D_{\kappa}\right|\right)+\sum_{\zeta= \pm \ell_{*}}\left(\left|C_{\zeta}\right|+\left|D_{\zeta, 1}\right|+\left|D_{\zeta, 2}\right|+\left|D_{\zeta, 3}\right|\right)\right)^{3}+ \\
\left(\sum_{\kappa \in \widetilde{I}_{*}}\left(\left|C_{\kappa}\right|+\left|D_{\kappa}\right|\right)+\sum_{\zeta= \pm \ell_{*}}\left(\left|C_{\zeta}\right|+\left|D_{\zeta, 1}\right|+\left|D_{\zeta, 2}\right|+\left|D_{\zeta, 3}\right|\right)\right)^{5} .
\end{gathered}
$$

\subsection{Existence of heteroclinic orbits}

We look for solutions of the system (3.14) in the form

$$
\begin{aligned}
C_{\ell}(x) & =\mathrm{e}^{\mathrm{i} \tilde{k}_{\ell, \mu, \delta} x} \widetilde{C_{\ell}}, \quad D_{\ell}(x)=\mathrm{e}^{\mathrm{i} \tilde{k}_{\ell, \mu, \delta} x} \widetilde{D_{\ell}}, \quad \ell \in I_{*} \\
C_{\ell_{*}}(x) & =\widetilde{C_{\ell_{*}}}(x), \quad D_{\ell_{*}, j}(x)=\widetilde{D_{\ell_{*}, j}}(x), \quad j=1,2,3 .
\end{aligned}
$$


With the scaling

$$
\begin{gathered}
\widehat{x}=|\mu|^{1 / 2} x, \quad \widetilde{C_{\ell}}=|\mu|^{1 / 2} \widehat{C_{\ell}}, \quad \widetilde{D_{\ell}}=|\mu| \widehat{D_{\ell}}, \quad \ell \in \widetilde{I}_{*}, \\
\widetilde{C_{\ell_{*}}}=|\mu|^{1 / 2} \widehat{C_{\ell_{*}}}, \quad \widetilde{D_{\ell_{*}, 1}}=|\mu|^{1 / 2} \widehat{D_{\ell_{*}, 1}}, \\
\widetilde{D_{\ell_{*}, 2}}=|\mu|^{1 / 2} \widehat{D_{\ell_{*}, 2}}, \quad \widetilde{D_{\ell_{*}, 3}}=|\mu|^{1 / 2} \widehat{D_{\ell_{*}, 3}},
\end{gathered}
$$

and taking $\mu>0$, we obtain the new system, for $\ell \in \widetilde{I}_{*}$,

$$
\begin{aligned}
C_{\ell}^{\prime} & =D_{\ell}+\mathcal{O}\left(\mu+\mu^{1 / 2}|\delta|\right) \\
D_{\ell}^{\prime} & =-\frac{1}{4 k_{x, \ell}^{2}} C_{\ell}\left(1-3\left(\left|C_{\ell_{*}}\right|^{2}-\left|C_{\ell}\right|^{2}+2 \sum_{\kappa \in I_{*}}\left|C_{\kappa}\right|^{2}\right)\right)+\mathcal{O}\left(\mu^{1 / 2}+|\delta|\right), \\
\mu^{1 / 2} C_{\ell_{*}}^{\prime} & =D_{\ell_{*}, 1}+\mathcal{O}\left(\mu^{2}\right) \\
\mu^{1 / 2} D_{\ell_{*}, 1}^{\prime} & =D_{\ell_{*}, 2}+\mathcal{O}\left(\mu^{2}\right) \\
\mu^{1 / 2} D_{\ell_{*}, 2}^{\prime} & =D_{\ell_{*}, 3}+\mathcal{O}\left(\mu^{2}\right) \\
\mu^{1 / 2} D_{\ell_{*}, 3}^{\prime} & =-2 k_{x, \ell_{*}, \delta}^{2} D_{\ell_{*}, 2}-k_{x, \ell_{*}, \delta}^{4} C_{\ell_{*}}+\mu C_{\ell_{*}}\left(1-3\left(\left|C_{\ell_{*}}\right|^{2}+2 \sum_{\kappa \in \widetilde{I}_{*}}\left|C_{\kappa}\right|^{2}\right)\right)+\mathcal{O}\left(\mu^{2}\right) .
\end{aligned}
$$

in which we have dropped the hats. Since the equations for $\left(C_{\ell_{*}}, D_{\ell_{*}, 1}, D_{\ell_{*}, 2}, D_{\ell_{*}, 3}\right)$ are singular, the strategy here is to break (3.15) up into a regular and a singular perturbation problem. The regular perturbation part

$$
C_{\ell}^{\prime \prime}=-\frac{1}{4 k_{x, \ell}^{2}} C_{\ell}+\frac{3}{4 k_{x, \ell}^{2}} C_{\ell}\left(\left|C_{\ell_{*}}\right|^{2}-\left|C_{\ell}\right|^{2}+2 \sum_{\kappa \in I_{*}}\left|C_{\kappa}\right|^{2}\right)+\mathcal{O}\left(\mu^{1 / 2}+|\delta|\right), \quad \ell \in I_{*},
$$

can be dealt with just as in the nonresonant case. As for the singular part, we rewrite it as follows.

$$
\begin{aligned}
\mu^{1 / 2} C_{\ell_{*}}^{\prime} & =D_{\ell_{*}, 1} \\
\mu^{1 / 2} D_{\ell^{\prime}, 1}^{\prime} & =D_{\ell_{*}, 2} \\
\mu^{1 / 2} D_{\ell_{*}, 2}^{\prime} & =D_{\ell_{*}, 3} \\
\mu^{1 / 2} D_{\ell_{*}, 3}^{\prime} & =-2 k_{x, \ell_{*}, \delta}^{2} D_{\ell_{*}, 2}-k_{x, \ell_{*}, \delta}^{4} C_{\ell_{*}}+\mu C_{\ell_{*}}\left(1-3\left(\left|C_{\ell_{*}}\right|^{2}+2 \sum_{\kappa \in \widetilde{I}_{*}}\left|C_{\kappa}\right|^{2}\right)\right)+\mathcal{O}\left(\mu^{2}\right),
\end{aligned}
$$

which can be achieved straightforwardly by an iterative family of smooth invertible coordinate changes.

Heteroclinic orbit of the leading order system Consider the system (3.16,3.17) with $\mu=0$ and $\delta=0$. Restricting to $C_{\ell}=0$ for $|\ell| \neq 1$ and real-valued solutions $C_{1}$ and $C_{-1}$ we find the system

$$
\begin{aligned}
C_{1}^{\prime \prime} & =-\frac{1}{4 k_{x, 1}^{2}} C_{1}+\frac{3}{4 k_{x, 1}^{2}} C_{1}\left(C_{1}^{2}+2 C_{-1}^{2}\right) \\
C_{-1}^{\prime \prime} & =-\frac{1}{4 k_{x, 1}^{2}} C_{-1}+\frac{3}{4 k_{x, 1}^{2}} C_{-1}\left(2 C_{1}^{2}+C_{-1}^{2}\right) .
\end{aligned}
$$

This system possesses a heteroclinic orbit $\left(C_{+}^{*}, C_{-}^{*}\right)$ with properties as those in the nonresonant case. 
The system (3.17) is a singular perturbation problem, which, viewed as an operator, is defined on different spaces when $(\mu, \delta) \neq(0,0)$ as compared to the case $(\mu, \delta)=(0,0)$. Our approach to this singular perturbation problem relies on the following lemma, which shows invertibility of the linearization uniformly for small $\mu \neq 0$.

Lemma 3.3 For fixed $\mu>0$ and fixed $\delta \in \mathbb{R}$, the linear operator

$$
\begin{aligned}
& \mathcal{S}:\left(H_{\eta}^{1}\right)^{4} \longrightarrow\left(L_{\eta}^{2}\right)^{4} \\
& \left(\begin{array}{l}
W_{1}(x) \\
W_{2}(x) \\
W_{3}(x) \\
W_{4}(x)
\end{array}\right) \longmapsto\left(\begin{array}{cccc}
\mu^{1 / 2} \partial_{x} & -1 & 0 & 0 \\
0 & \mu^{1 / 2} \partial_{x} & -1 & 0 \\
0 & 0 & \mu^{1 / 2} \partial_{x} & -1 \\
g(x)+k_{x, \ell_{*}, \delta}^{4} / \mu & 0 & 2 k_{x, \ell_{*}, \delta}^{2} / \mu & \mu^{-1 / 2} \partial_{x}
\end{array}\right)\left(\begin{array}{l}
W_{1}(x) \\
W_{2}(x) \\
W_{3}(x) \\
W_{4}(x)
\end{array}\right)
\end{aligned}
$$

where $g(x)=6\left(C_{+}^{* 2}+C_{-}^{* 2}\right)-1$, is bounded and invertible, for sufficiently small $\eta>0$. Moreover, for fixed $\eta>0$ small, and for $\mu>0, \delta \sim 0$ small, there exists a positive constant $C \geqslant 1$ independent of $\mu$ and $\delta$ such that

$$
\left\|\mathcal{S}^{-1}\left(\begin{array}{c}
0 \\
0 \\
0 \\
f
\end{array}\right)\right\|_{\left(H_{\eta}^{1}\right)^{4}} \leqslant C\|f\|_{H_{\eta}^{1}}
$$

for all $f \in H_{\eta}^{1}$.

Proof. We define the equivalent scalar fourth-order differential operator

$$
\begin{aligned}
\widetilde{\mathcal{S}}: H_{\eta}^{4} & \longrightarrow L_{\eta}^{2} \\
C(x) & \longmapsto \frac{1}{\mu}\left(\mu \partial_{x}^{2}+k_{x, \ell_{*}, \delta}^{2}\right)^{2} C(x)+g(x) C(x) .
\end{aligned}
$$

From [10], we know that $\mathcal{S}$ and $\widetilde{\mathcal{S}}$ share the same Fredholm index. Moreover, $\operatorname{Ker}(\widetilde{\mathcal{S}})=\{0\}$ if and only if $\operatorname{Ker}(\mathcal{S})=\{0\}$. Thus, it is enough to prove the same properties for $\widetilde{\mathcal{S}}$. It is straightforward to see that $\widetilde{\mathcal{S}}$ is bounded. To show that $\widetilde{\mathcal{S}}$ is invertible, we just need to show $\widetilde{\mathcal{S}_{\eta}}$ is invertible, where

$$
\begin{aligned}
\widetilde{\mathcal{S}_{\eta}}: H^{4} & \longrightarrow L^{2} \\
C(x) & \longmapsto \cosh (\eta x) \mathcal{S}\left(\frac{1}{\cosh (\eta x)} C(x)\right) .
\end{aligned}
$$

Note that $\widetilde{\mathcal{S}_{\eta}}$ is a family of smooth bounded operators with respect to $\eta$ and $\widetilde{\mathcal{S}_{\eta}}=\widetilde{\mathcal{S}_{0}}+\eta \cdot \mathcal{O}(1)$. Therefore, to show $\widetilde{\mathcal{S}_{\eta}}$ is invertible for sufficiently small $\eta$, we only have to show that $\widetilde{\mathcal{S}_{0}}$ is invertible. In fact, according to property $v$ of $\left(C_{+}^{*}, C_{-}^{*}\right)$,

$$
\frac{1}{6} \leqslant C_{+}^{* 2}+C_{-}^{* 2} \leqslant \frac{1}{3}
$$

where the left equality holds if and only if $\left(C_{+}^{*}, C_{-}^{*}\right)=\left(\frac{1}{2 \sqrt{3}}, \frac{1}{2 \sqrt{3}}\right)$. If the curve $\left(C_{+}^{*}, C_{-}^{*}\right)$ touches $\left(\frac{1}{2 \sqrt{3}}, \frac{1}{2 \sqrt{3}}\right)$, by properties (iii,iv), $C_{+}^{*}+C_{-}^{*}$ is an even function and $C_{+}^{*}(0)+C_{-}^{*}(0)=\frac{1}{\sqrt{3}}$. Moreover, according to system (2.27), we have

$$
\left.\left(C_{+}^{*}+C_{-}^{*}\right)^{\prime \prime}\right|_{x=0}=-\frac{1}{4 \sqrt{3}}<0 .
$$


As a result, for sufficiently small $|x| \neq 0, C_{+}^{*}(x)+C_{-}^{*}(x)<\frac{1}{\sqrt{3}}$, which is a contradiction with property (v). Thus, there exists a positive constant $\xi$ such that

$$
\frac{1}{6}+\xi \leqslant C_{+}^{* 2}+C_{-}^{* 2} \leqslant \frac{1}{3}
$$

Now we look into $\widetilde{\mathcal{S}}_{0}$, which is self-adjoint and

$$
\left\langle\widetilde{\mathcal{S}}_{0} f, f\right\rangle_{L^{2}} \geqslant 6 \xi\|f\|_{L^{2}}^{2},
$$

which indicates that, for sufficiently small $\mu$ and $\delta, \mathcal{S}_{0}$ is invertible and

$$
\left\|\left(\widetilde{\mathcal{S}}_{0}\right)^{-1} f\right\|_{L^{2}} \leqslant \frac{1}{6 \xi}\|f\|_{L^{2}}
$$

which shows that $\mathcal{S}(\mu, \delta)$ is bounded and invertible for sufficiently small $\eta>0$. In addition, there exists some positive constant $N(\xi)$ such that

$$
\left\|(\widetilde{\mathcal{S}}(\mu, \delta))^{-1} f\right\|_{H_{\eta}^{1}} \leqslant N\|f\|_{H_{\eta}^{1}} .
$$

In fact, we have

$$
\partial_{x}\left(\widetilde{\mathcal{S}}^{-1} f\right)=\left[\partial_{x}, \widetilde{\mathcal{S}}^{-1}\right] f+\widetilde{\mathcal{S}}^{-1}\left(\partial_{x} f\right)=\widetilde{\mathcal{S}}^{-1}\left[\widetilde{\mathcal{S}}, \partial_{x}\right] \widetilde{\mathcal{S}}^{-1} f+\widetilde{\mathcal{S}}^{-1}\left(\partial_{x} f\right)
$$

where $\left[\widetilde{\mathcal{S}}, \partial_{x}\right]=g^{\prime}(x)$. This new expression (3.24), combined with the $L_{\eta}^{2}$ estimate $(3.22)$, gives the $H_{\eta}^{1}$ estimate in (3.23). We now notice that

$$
\mathcal{S}^{-1}\left(\begin{array}{l}
0 \\
0 \\
0 \\
f
\end{array}\right)=\left(\begin{array}{c}
\widetilde{\mathcal{S}}^{-1} f \\
\mu^{1 / 2} \partial_{x} \widetilde{\mathcal{S}}^{-1} f \\
\mu \partial_{x}^{2} \widetilde{\mathcal{S}}^{-1} f \\
\mu^{3 / 2} \partial_{x}^{3} \widetilde{\mathcal{S}}^{-1} f
\end{array}\right)
$$

which, combined with (3.24), shows that there exists some $N \geqslant 1$ such that

$$
\left\|\mathcal{S}^{-1}\left(\begin{array}{c}
0 \\
0 \\
0 \\
f
\end{array}\right)\right\| \|_{\left(H_{\eta}^{1}\right)^{4}} \leqslant N \sum_{j=0}^{3} \mu^{j / 2}\left(\left\|\partial_{x}^{j}(\widetilde{\mathcal{S}})^{-1} f\right\|_{L_{\eta}^{2}}+\left\|\partial_{x}^{j}(\widetilde{\mathcal{S}})^{-1}\left(g(x) \widetilde{\mathcal{S}}^{-1} f\right)\right\|_{L_{\eta}^{2}}+\left\|\partial_{x}^{j}(\widetilde{\mathcal{S}})^{-1} \partial_{x} f\right\|_{L_{\eta}^{2}}\right) .
$$

By the inequality (3.26), in order to prove that (3.20) holds for all $f \in H_{\eta}^{1}$, we only have to show that for all $f \in L_{\eta}^{2}$, there exists a positive constant $N \geqslant 1$ such that

$$
\begin{gathered}
\left\|\partial_{x}(\widetilde{\mathcal{S}}(\mu, \delta))^{-1} f\right\|_{L_{\eta}^{2}} \leqslant \frac{N}{\mu^{1 / 2}}\|f\|_{L_{\eta}^{2}}, \\
\left\|\partial_{x}^{2}(\widetilde{\mathcal{S}}(\mu, \delta))^{-1} f\right\|_{L_{\eta}^{2}} \leqslant \frac{N}{\mu}\|f\|_{L_{\eta}^{2}}, \\
\left\|\partial_{x}^{3}(\widetilde{\mathcal{S}}(\mu, \delta))^{-1} f\right\|_{L_{\eta}^{2}} \leqslant \frac{N}{\mu^{3 / 2}}\|f\|_{L_{\eta}^{2}} .
\end{gathered}
$$

We relegate the proof of these inequalities to the appendix, Lemma 4.1. 
For sufficiently small $\mu \geqslant 0$ and $\delta \in \mathbb{R}$, we now rewrite the system $(3.16,3.17)$ as follows.

$$
\begin{gathered}
C_{\ell}^{\prime \prime}+\frac{1}{4 k_{x, \ell}^{2}} C_{\ell}-\frac{3}{4 k_{x, \ell}^{2}} C_{\ell}\left(\left|C_{\ell_{*}}\right|^{2}-\left|C_{\ell}\right|^{2}+2 \sum_{\kappa \in I_{*}}\left|C_{\kappa}\right|^{2}\right)+\mathcal{O}\left(\mu^{1 / 2}+|\delta|\right)=0, \quad \ell \in \widetilde{I}_{*} \\
\mathcal{S}(\mu, \delta)\left(\begin{array}{c}
C_{\ell_{*}} \\
D_{\ell_{*}, 1} \\
D_{\ell_{*}, 2} \\
D_{\ell_{*}, 3}
\end{array}\right)+\left(\begin{array}{c}
0 \\
0 \\
0 \\
f(x)
\end{array}\right)=0,
\end{gathered}
$$

where $f(x)=3 C_{\ell_{*}}\left(\left|C_{\ell_{*}}\right|^{2}+2 \sum_{\kappa \in \widetilde{I}_{*}}\left|C_{\kappa}\right|^{2}-2 C_{+}^{* 2}-2 C_{-}^{* 2}\right)+\mathcal{O}(|\mu|)$.

Rotated rolls. Similar to the non-resonant case, the family of rolls in (2.25) gives a family of periodic orbits for (3.28,3.29), still denoted as $\mathbf{P}_{\mu, \delta, \varepsilon, \ell}$ and $\mathbf{Q}_{\mu, \delta, \varepsilon, \ell}$ for all $\ell \in \widetilde{I}_{*}$. Again, we just consider $\ell=1$.

Persistence of the heteroclinic orbit Our main goal now is to show that the heteroclinic actually persists as a solution for the reduced equation (3.28,3.29). In particular, we want to show that there is a heteroclinic orbit for $(3.28,3.29)$, for small $\mu$ and $\delta$, which connects two periodic orbits $\mathbf{P}_{\mu, \delta, \varepsilon, 1}$, as $x \rightarrow \infty$, and $\mathbf{Q}_{\mu, \delta, \varepsilon, 1}$, as $x \rightarrow-\infty$.

The systems (3.28) and (3.29) together with the complex conjugated equations are separately of the form

$$
\begin{array}{r}
\mathcal{F}_{1}\left(\mathbf{C}, \overline{\mathbf{C}}, \mu^{1 / 2}, \delta\right)=0 \\
\mathcal{F}_{2}\left(\mathbf{C}, \overline{\mathbf{C}}, \mu^{1 / 2}, \delta\right)=0
\end{array}
$$

where

$$
\begin{array}{r}
\mathbf{C}=\left(C_{-\left(\ell_{*}-1\right)}, \ldots, C_{-2}, C_{-1}, C_{0}, C_{1}, C_{2}, \ldots, C_{\ell_{*}}, D_{\ell_{*}, 1}, D_{\ell_{*}, 2}, D_{\ell_{*}, 3}\right), \\
\widetilde{\mathbf{C}}=\left(C_{-\left(\ell_{*}-1\right)}, \ldots, C_{-2}, C_{-1}, C_{0}, C_{1}, C_{2}, \ldots, C_{\ell_{*}-1}\right) .
\end{array}
$$

They have the periodic solutions $\mathbf{C}=\mathbf{P}_{\mu, \delta, \varepsilon, 1}$ and $\mathbf{C}=\mathbf{Q}_{\mu, \delta, \varepsilon, 1}$ for $\mu, \delta$ and $\varepsilon$ sufficiently small, and the heteroclinic solution $\mathbf{C}=\left(0, \ldots, 0, C_{-}^{*}, 0, C_{+}^{*}, 0, \ldots, 0\right)$ for $\mu=\delta=0$.

We consider the ansatz

$$
\mathbf{C}(x)=\mathrm{e}^{\mathrm{i} \varepsilon x} \mathbf{C}^{*}(x)+\chi(x) \widetilde{\mathbf{P}}_{\mu, \delta, \varepsilon, 1}(x)+\left(\mathcal{R}\left(\chi \widetilde{\mathbf{P}}_{\mu, \delta, \varepsilon, 1}\right)\right)(-x)+\mathbf{V}(x),
$$

in which heteroclinic orbit and correction to the periodic orbit are given as

$$
\mathbf{C}^{*}=\left(0, \ldots, 0, C_{-}^{*}, 0, C_{+}^{*}, 0, \ldots, 0\right), \quad \widetilde{\mathbf{P}}_{\mu, \delta, \varepsilon, 1}=\mathbf{P}_{\mu, \delta, \varepsilon, 1}-\left(0, \ldots, 0,0,0, \frac{1}{\sqrt{3}}, 0, \ldots, 0\right) \mathrm{e}^{\mathrm{i} \varepsilon x},
$$

$\chi: \mathbb{R} \rightarrow[0,1]$ is a smooth cut-off function with

$$
\chi(x)=1, \text { if } x \geqslant M, \quad \chi(x)=0, \text { if } x \leqslant m, \quad \chi(x)+\chi(-x) \equiv 1,
$$

for some positive constants $m<M$. Moreover, we have

$$
\mathbf{V}=(\widetilde{\mathbf{V}}, \mathbf{W}), \quad \tilde{\mathbf{V}}=\left(V_{-\left(\ell_{*}-1\right)}, \ldots, V_{-1}, V_{0}, V_{1}, \ldots, V_{\ell_{*}-1}\right), \quad \mathbf{W}=\left(V_{\ell_{*}}, V_{\ell_{*}, 1}, V_{\ell_{*}, 2}, V_{\ell_{*}, 3}\right)
$$


We next modify $(3.30,3.31)$, by adding zero terms, where the functions $\mathcal{F}_{j}$ are evaluated on the periodicorbit solutions, in order to make mapping properties in exponentially weighted spaces more explicit later on. Therefore, define

$$
\begin{gathered}
\mathcal{F}_{1}\left(\mathbf{C}, \overline{\mathbf{C}}, \mu^{1 / 2}, \delta\right)-\chi(x) F_{1}\left(\mathbf{P}_{\mu, \delta, \varepsilon, 1}, \overline{\mathbf{P}_{\mu, \delta, \varepsilon, 1}}, \mu^{1 / 2}, \delta\right)-\chi(-x) F_{1}\left(\mathbf{Q}_{\mu, \delta, \varepsilon, 1}, \overline{\mathbf{Q}_{\mu, \delta, \varepsilon, 1}}, \mu^{1 / 2}, \delta\right)=0 \\
\mathcal{F}_{2}\left(\mathbf{C}, \overline{\mathbf{C}}, \mu^{1 / 2}, \delta\right)-\chi(x) F_{2}\left(\mathbf{P}_{\mu, \delta, \varepsilon, 1}, \overline{\mathbf{P}_{\mu, \delta, \varepsilon, 1}}, \mu^{1 / 2}, \delta\right)-\chi(-x) F_{2}\left(\mathbf{Q}_{\mu, \delta, \varepsilon, 1}, \overline{\mathbf{Q}_{\mu, \delta, \varepsilon, 1}}, \mu^{1 / 2}, \delta\right)=0,
\end{gathered}
$$

and substitute our ansatz (3.32) into $(3.33,3.34)$, to obtain a system of the form

$$
\begin{aligned}
& \mathcal{T}_{1}\left(\tilde{\mathbf{V}}, \overline{\mathbf{V}}, \mathbf{W}, \overline{\mathbf{W}}, \varepsilon, \mu^{1 / 2}, \delta\right)=0, \\
& \mathcal{T}_{2}\left(\tilde{\mathbf{V}}, \overline{\widetilde{\mathbf{V}}}, \mathbf{W}, \overline{\mathbf{W}}, \varepsilon, \mu^{1 / 2}, \delta\right)=0 .
\end{aligned}
$$

For $\eta>0$, we define the spaces of exponentially decaying functions

$$
\begin{array}{r}
\widetilde{\mathcal{X}_{\eta}}=\left\{\left(C_{\ell}, \overline{C_{\ell}}\right)_{\ell \in \widetilde{I_{*}}} \in\left(L_{\eta}^{2}\right)^{4 \ell_{*}-2}\right\}, \quad \widetilde{\mathcal{Y}_{\eta}}=\left\{\left(C_{\ell}, \overline{C_{\ell}}\right)_{\ell \in \widetilde{I_{*}}} \in\left(H_{\eta}^{2}\right)^{4 \ell_{*}-2}\right\}, \\
\widetilde{\mathcal{X}_{\eta}^{r}}=\left\{\left(C_{\ell}, \overline{C_{\ell}}\right)_{\ell \in \widetilde{I_{*}}} \in \widetilde{\mathcal{X}_{\eta}} ; C_{\ell}(x)=\overline{C_{-\ell}}(-x), x \in \mathbb{R}, \ell \in \widetilde{I_{*}}\right\}, \\
\widetilde{\mathcal{Y}_{\eta}^{r}}=\left\{\left(C_{\ell}, \overline{C_{\ell}}\right)_{\ell \in \widetilde{I_{*}}} \in \widetilde{\mathcal{Y}}_{\eta} ; C_{\ell}(x)=\overline{C_{-\ell}}(-x), x \in \mathbb{R}, \ell \in \widetilde{I_{*}}\right\}, \\
\mathcal{Z}_{\eta}^{r}=\left\{\left(W_{j}, \overline{W_{j}}\right)_{j=1,2,3,4} \in\left(H_{\eta}^{1}\right)^{8} \mid W_{j}(x)=W_{j}(-x), j=1,2,3,4\right\}, \\
\widetilde{\mathcal{Z}_{\eta}^{r}}=\left\{\left(W_{j}, \overline{W_{j}}\right)_{j=1,2,3,4} \in\left(L_{\eta}^{2}\right)^{8} \mid W_{j}(x)=W_{j}(-x), j=1,2,3,4\right\},
\end{array}
$$

It is now not hard to verify that

$$
\begin{array}{ll}
\mathcal{T}_{1}: \widetilde{\mathcal{Y}_{\eta}^{r}} \times \mathcal{Z}_{\eta}^{r} \times \mathbb{R}^{3} & \longrightarrow \widetilde{\mathcal{X}_{\eta}^{r}}, \\
\mathcal{T}_{2}: \widetilde{\mathcal{Y}_{\eta}^{r}} \times \mathcal{Z}_{\eta}^{r} \times \mathbb{R}^{3} \longrightarrow \widetilde{\mathcal{Z}_{\eta}^{r}}
\end{array}
$$

are well-defined nonlinear operators. We start by solving the first of these two equations, which is in fact similar to the non-resonant case.

Proposition 3.4 For $(\mathbf{W}, \overline{\mathbf{W}}, \mu, \delta) \in \mathcal{Z}_{\eta}^{r} \times[0, \infty) \times \mathbb{R}$ sufficiently small, there exist smooth functions $\widetilde{\mathbf{V}}\left(\mathbf{W}, \overline{\mathbf{W}}, \mu^{1 / 2}, \delta\right)$ and $\varepsilon\left(\mathbf{W}, \overline{\mathbf{W}}, \mu^{1 / 2}, \delta\right)$, solving equation $(3.35)$, with $\widetilde{\mathbf{V}}(0,0,0,0)=0$ and $\varepsilon(0,0,0,0)=$ 0 .

Proof. The proof is based on the inverse-function-theorem argument from the nonresonant case. A key role is played by the linear operator, found by linearizing the system (3.28) at

$$
\left(C_{-\left(\ell_{*}-1\right)}, \ldots, C_{-2}, C_{-1}, C_{0}, C_{1}, C_{2}, \ldots, C_{\ell_{*}-1}\right)=\left(0, \ldots, 0, C_{-}^{*}, 0, C_{+}^{*}, 0, \ldots, 0\right),
$$

with $\mu=\delta=0$ and $C_{\ell_{*}}=D_{\ell_{*}, 1}=D_{\ell_{*}, 2}=D_{\ell_{*}, 3}=0$, i.e.,

$$
\widetilde{\mathcal{L}_{*}}\left(\begin{array}{c}
C_{-\left(\ell_{*}-1\right)} \\
\ldots \\
C_{-2} \\
C_{-1} \\
C_{0} \\
C_{1} \\
C_{2} \\
\ldots \\
C_{\ell_{*}-1}
\end{array}\right)=\left(\begin{array}{c}
C_{-\left(\ell_{*}-1\right)}^{\prime \prime}+\frac{1}{4 k_{x,-\left(\ell_{*}-1\right)}^{2}} C_{-\left(\ell_{*}-1\right)}-\frac{3}{2 k_{x,-\left(\ell_{*}-1\right)}^{2}}\left(C_{+}^{* 2}+C_{-}^{* 2}\right) C_{-\left(\ell_{*}-1\right)} \\
C_{-2}^{\prime \prime}+\frac{1}{4 k_{x,-2}^{2}} C_{-2}-\frac{3}{2 k_{x,-2}^{2}}\left(C_{+}^{* 2}+C_{-}^{* 2}\right) C_{-2} \\
C_{-1}^{\prime \prime}+\frac{1}{4 k_{x, 1}^{2}} C_{-1}-\frac{3}{4 k_{x, 1}^{2}}\left(2\left(C_{+}^{* 2}+C_{-}^{* 2}\right) C_{-1}+C_{-}^{* 2} \overline{C_{-1}}+2 C_{+}^{*} C_{-}^{*}\left(C_{1}+\overline{C_{1}}\right)\right) \\
C_{0}^{\prime \prime}+\frac{1}{4 k_{x, 0}^{2}} C_{\ell}-\frac{3}{2 k_{x, 0}^{2}}\left(C_{+}^{* 2}+C_{-}^{* 2}\right) C_{0} \\
C_{1}^{\prime \prime}+\frac{1}{4 k_{x, 1}^{2}} C_{1}-\frac{3}{4 k_{x, 1}^{2}}\left(2\left(C_{+}^{* 2}+C_{-}^{* 2}\right) C_{1}+C_{+}^{* 2} \overline{C_{1}}+2 C_{+}^{*} C_{-}^{*}\left(C_{-1}+\overline{C_{-1}}\right)\right) \\
C_{2}^{\prime \prime}+\frac{1}{4 k_{x, 2}^{2}} C_{2}-\frac{3}{2 k_{x, 2}^{2}}\left(C_{+}^{* 2}+C_{-}^{* 2}\right) C_{2} \\
\cdots \\
C_{\ell_{*}-1}^{\prime \prime}+\frac{1}{4 k_{x, \ell_{*}-1}^{2}} C_{\ell_{*}-1}-\frac{3}{2 k_{x, \ell_{*}-1}^{2}}\left(C_{+}^{* 2}+C_{-}^{* 2}\right) C_{\ell_{*}-1}
\end{array}\right) .
$$


For $\eta>0$ sufficiently small, the operator $\widetilde{\mathcal{L}_{*}}$ acting in $\widetilde{\mathcal{X}_{\eta}^{r}}$ is Fredholm with trivial kernel and onedimensional co-kernel, spanned by

$$
\left(0, \ldots, 0,-\mathrm{i} C_{-}^{*}, 0, \mathrm{i} C_{+}^{*}, 0, \ldots, 0 ; 0, \ldots, 0, \mathrm{i} C_{-}^{*}, 0,-\mathrm{i} C_{+}^{*}, 0, \ldots, 0\right)
$$

Next, notice that

$$
\mathcal{T}_{1}(0,0,0,0,0,0,0)=0, \quad D_{\widetilde{\mathbf{V}}, \widetilde{\mathbf{V}}} \mathcal{T}_{1}(0,0,0,0,0,0,0)=\widetilde{\mathcal{L}_{*}}
$$

and

$$
D_{\varepsilon} \mathcal{T}(0,0,0,0,0,0,0)=\widetilde{\mathcal{L}_{*}}\left(\begin{array}{c}
i x \widetilde{\mathbf{C}^{*}} \\
-i x \widetilde{\mathbf{C}^{*}}
\end{array}\right)=\left(\begin{array}{c}
2 i \widetilde{\mathbf{C}^{* \prime}} \\
-2 i \widetilde{\mathbf{C}^{* \prime}}
\end{array}\right)
$$

Using the explicit form of the co-kernel, we find that the linearization with respect to $\widetilde{\mathbf{V}}, \tilde{\mathbf{V}}$ and $\varepsilon$, jointly, is invertible, so that we can use the implicit function theorem to conclude persistence as stated in the theorem.

We substitute the solutions $\widetilde{\mathbf{V}}\left(\mathbf{W}, \overline{\mathbf{W}}, \mu^{1 / 2}, \delta\right)$ and $\varepsilon\left(\mathbf{W}, \overline{\mathbf{W}}, \mu^{1 / 2}, \delta\right)$ from Proposition 3.4 into (3.36) and obtain

$$
\mathcal{T}_{3}\left(\mathbf{W}, \overline{\mathbf{W}}, \mu^{1 / 2}, \delta\right)=0
$$

Theorem 3 For any $\mu$ and $\delta$ sufficiently small, there exists continuous function $\mathbf{W}\left(\mu^{1 / 2}, \delta\right)$ solving equation (3.37), with $\mathbf{W}(0,0)=0$.

Proof. We define the map

$$
\begin{aligned}
\mathcal{T}_{4}: \mathcal{Z}_{\eta}^{r} \times[0, \infty) \times \mathbb{R} & \longrightarrow \mathcal{Z}_{\eta}^{r} \\
\left(\mathbf{W}, \overline{\mathbf{W}}, \mu^{1 / 2}, \delta\right) & \longmapsto(\mathbf{W}, \overline{\mathbf{W}})-(\mathcal{S}(\mu, \delta))^{-1} \mathcal{T}_{3}\left(\mathbf{W}, \overline{\mathbf{W}}, \mu^{1 / 2}, \delta\right),
\end{aligned}
$$

and extend to $\mu=0$ by setting

$$
(\mathcal{S}(0, \delta))^{-1}:=0
$$

To prove the theorem, we only have to show that there exist two small neighborhoods $\mathcal{W}=\{(\mathbf{W}, \overline{\mathbf{W}}) \in$ $\mathcal{Z}_{\eta}^{r} \mid \|\left(\mathbf{W}, \overline{\mathbf{W}} \|_{\mathcal{Z}_{\eta}^{r}}<a\right\}$ of $(\mathbf{W}, \overline{\mathbf{W}})=(0,0)$ and $\mathcal{U} \subset[0, \infty) \times \mathbb{R}$ of $\left(\mu^{1 / 2}, \delta\right)=(0,0)$ such that $\mathcal{T}_{4}:$ $\overline{\mathcal{W}} \times \mathcal{U} \longrightarrow \overline{\mathcal{W}}$ is a uniform contraction. We only have to show that $\mathcal{T}_{4}$ has the following properties.

(i) $\left\|\mathcal{T}_{4}\left(\mathbf{W}, \overline{\mathbf{W}}, \mu^{1 / 2}, \delta\right)\right\|_{\mathcal{Z}_{\eta}^{r}} \leqslant a$, for all $(\mathbf{W}, \overline{\mathbf{W}}) \in \overline{\mathcal{W}}$ and $\left(\mu^{1 / 2}, \delta\right) \in \mathcal{U}$.

(ii)

$$
\left\|\mathcal{T}_{4}\left(\mathbf{W}_{1}, \overline{\mathbf{W}_{1}}, \mu^{1 / 2}, \delta\right)-\mathcal{T}_{4}\left(\mathbf{W}_{2}, \overline{\mathbf{W}_{2}}, \mu^{1 / 2}, \delta\right)\right\|_{\mathcal{Z}_{\eta}^{r}} \leqslant \frac{1}{2}\left\|\left(\mathbf{W}_{1}, \overline{\mathbf{W}_{1}}\right)-\left(\mathbf{W}_{2}, \overline{\mathbf{W}_{2}}\right)\right\|_{\mathcal{Z}_{\eta}^{r}}
$$

for all $\left(\mathbf{W}_{1}, \overline{\mathbf{W}_{1}}\right),\left(\mathbf{W}_{2}, \overline{\mathbf{W}_{2}}\right) \in \mathcal{W}$ and $\left(\mu^{1 / 2}, \delta\right) \in \mathcal{U}$.

To see that, we first recall that equation (3.37) is equation (3.34)

$$
\mathcal{F}_{2}\left(\mathbf{C}, \overline{\mathbf{C}}, \mu^{1 / 2}, \delta\right)-\chi(x) F_{2}\left(\mathbf{P}_{\mu, \delta, \varepsilon, 1}, \overline{\mathbf{P}_{\mu, \delta, \varepsilon, 1}}, \mu^{1 / 2}, \delta\right)-\chi(-x) F_{2}\left(\mathbf{Q}_{\mu, \delta, \varepsilon, 1}, \overline{\mathbf{Q}_{\mu, \delta, \varepsilon, 1}}, \mu^{1 / 2}, \delta\right)=0
$$


together with its conjugate in terms of $\left(\mathbf{W}, \overline{\mathbf{W}}, \mu^{1 / 2}, \delta\right)$. Moreover, we recall that

$$
\mathcal{F}_{2}\left(\mathbf{C}, \overline{\mathbf{C}}, \mu^{1 / 2}, \delta\right)=\mathcal{S}(\mu, \delta)\left(\begin{array}{c}
C_{\ell_{*}} \\
D_{\ell_{*}, 1} \\
D_{\ell_{*}, 2} \\
D_{\ell_{*}, 3}
\end{array}\right)+\left(\begin{array}{c}
0 \\
0 \\
0 \\
f(x)
\end{array}\right),
$$

in which $f(x)=3 C_{\ell_{*}}\left(\left|C_{\ell_{*}}\right|^{2}+2 \sum_{\kappa \in \widetilde{I}_{*}}\left|C_{\kappa}\right|^{2}-2 C_{+}^{* 2}-2 C_{-}^{* 2}\right)+\mathcal{O}(|\mu|)$ and that $\mathcal{O}(\mu)$ here represents the higher order terms of order

$$
\mu\left(\sum_{\kappa \in \widetilde{I}_{*}}\left(\left|C_{\kappa}\right|+\left|D_{\kappa}\right|\right)+\sum_{\zeta= \pm \ell_{*}}\left(\left|C_{\zeta}\right|+\left|D_{\zeta, 1}\right|+\left|D_{\zeta, 2}\right|+\left|D_{\zeta, 3}\right|\right)\right)^{3} .
$$

A lengthy calculation shows that

$$
\mathcal{T}_{4}\left(\mathbf{W}, \overline{\mathbf{W}}, \mu^{1 / 2}, \delta\right)=\left(\begin{array}{l}
U\left(\varepsilon, \mu^{1} / 2, \delta\right) \\
\bar{U}\left(\varepsilon, \mu^{1} / 2, \delta\right)
\end{array}\right)+\left(\begin{array}{c}
(0 \\
(\mathcal{S}(\mu, \delta))^{-1}\left(\begin{array}{c}
(0) \\
0 \\
\widetilde{f})
\end{array}\right) \\
(\mathcal{S}(\mu, \delta))^{-1}\left(\begin{array}{c}
(0 \\
0 \\
0 \\
\widetilde{\widetilde{f}})
\end{array}\right)
\end{array}\right)
$$

in which

$$
\|(U, \bar{U})\|_{\mathcal{Z}_{\eta}^{r}}=\mathcal{O}\left(\mu^{1 / 2}\right), \quad\|\widetilde{f}\|_{H_{\eta}^{1}}=\mathcal{O}\left(\|(\mathbf{W}, \overline{\mathbf{W}})\|_{\mathcal{Z}_{\eta}^{r}}^{2}+\left(\mu^{1 / 2}+|\delta|\right)\|(\mathbf{W}, \overline{\mathbf{W}})\|_{\mathcal{Z}_{\eta}^{r}}+\mu^{1 / 2}\right) .
$$

We recall the estimate (3.20) in Lemma 3.3,

$$
\left\|\mathcal{S}^{-1}\left(\begin{array}{c}
0 \\
0 \\
0 \\
f
\end{array}\right)\right\|_{\left(H_{\eta}^{1}\right)^{4}} \leqslant C\|f\|_{H_{\eta}^{1}}
$$

which, combined with (3.41), shows that there exist positive constants $C_{1}, C_{2} \geqslant 1$ such that

$$
\left\|\mathcal{T}_{4}\left(\mathbf{W}, \overline{\mathbf{W}}, \mu^{1 / 2}, \delta\right)\right\|_{\mathcal{Z}_{\eta}^{r}} \leqslant C_{1}\|(\mathbf{W}, \overline{\mathbf{W}})\|_{\mathcal{Z}_{\eta}^{r}}^{2}+\left(\mu^{1 / 2}+|\delta|\right) C_{1}\|(\mathbf{W}, \overline{\mathbf{W}})\|_{\mathcal{Z}_{\eta}^{r}}+C_{2} \mu^{1 / 2}
$$

Noting that $h(x)=C_{1} x^{2}+\left(\mu^{1 / 2}+\delta\right) C_{1} x+C_{2} \mu^{1 / 2}$ is positive and increasing on $[0, \infty)$ and solving the inequality $C_{1} a^{2}+\left(\mu^{1 / 2}+\delta\right) C_{1} a+C_{2} \mu^{1 / 2} \leqslant a$, we conclude that property (i) of $\mathcal{T}_{4}$ holds as long as we take

$$
\mu^{1 / 2} \leqslant \frac{1}{16 C_{1} C_{2}}, \quad|\delta| \leqslant \frac{7}{16 C_{1} C_{2}}, \quad a=\frac{1-C_{1}\left(\mu^{1 / 2}+|\delta|\right)-\sqrt{\left(1-C_{1}\left(\mu^{1 / 2}+|\delta|\right)\right)^{2}-4 C_{1} C_{2} \mu^{1 / 2}}}{2 C_{1}} .
$$

On the other hand, there exist positive constants $C_{3}, C_{4} \geqslant 1$ such that

$$
\begin{aligned}
\left\|\mathcal{T}_{4}\left(\mathbf{W}_{1}, \overline{\mathbf{W}_{1}}, \mu^{1 / 2}, \delta\right)-\mathcal{T}_{4}\left(\mathbf{W}_{2}, \overline{\mathbf{W}_{2}}, \mu^{1 / 2}, \delta\right)\right\|_{\mathcal{Z}_{\eta}^{r}} \leqslant C_{3} \mu^{1 / 2}\left\|\left(\mathbf{W}_{1}, \overline{\mathbf{W}_{1}}\right)-\left(\mathbf{W}_{2}, \overline{\mathbf{W}_{2}}\right)\right\|_{\mathcal{Z}_{\eta}^{r}+} \\
C_{4}\left\|\widetilde{f}\left(\mathbf{W}_{1}, \overline{\mathbf{W}_{1}}, \mu^{1 / 2}, \delta\right)-\widetilde{f}\left(\mathbf{W}_{2}, \overline{\mathbf{W}_{2}}, \mu^{1 / 2}, \delta\right)\right\|_{L_{\eta}^{2}} .
\end{aligned}
$$


A straightforward calculation shows that there exists a positive constant $C_{5} \geqslant 1$ such that

$$
\left\|\widetilde{f}\left(\mathbf{W}_{1}, \overline{\mathbf{W}_{1}}, \mu^{1 / 2}, \delta\right)-\widetilde{f}\left(\mathbf{W}_{2}, \overline{\mathbf{W}_{2}}, \mu^{1 / 2}, \delta\right)\right\|_{H_{\eta}^{1}} \leqslant C_{5}\left(a+\mu^{1 / 2}+|\delta|\right)\left\|\left(\mathbf{W}_{1}, \overline{\mathbf{W}_{1}}\right)-\left(\mathbf{W}_{2}, \overline{\mathbf{W}_{2}}\right)\right\|_{\mathcal{Z}_{\eta}^{r}},
$$

which, plugged into equation (3.43), gives that

$$
\begin{aligned}
& \left\|\mathcal{T}_{4}\left(\mathbf{W}_{1}, \overline{\mathbf{W}_{1}}, \mu^{1 / 2}, \delta\right)-\mathcal{T}_{4}\left(\mathbf{W}_{2}, \overline{\mathbf{W}_{2}}, \mu^{1 / 2}, \delta\right)\right\|_{\mathcal{Z}_{\eta}^{r}} \\
\leqslant & \left(C_{3} \mu^{1 / 2}+C_{4} C_{5}\left(a+\mu^{1 / 2}+|\delta|\right)\right)\left\|\left(\mathbf{W}_{1}, \overline{\mathbf{W}_{1}}\right)-\left(\mathbf{W}_{2}, \overline{\mathbf{W}_{2}}\right)\right\|_{\mathcal{Z}_{\eta}^{r}} \\
\leqslant & \frac{1}{2}\left\|\left(\mathbf{W}_{1}, \overline{\mathbf{W}_{1}}\right)-\left(\mathbf{W}_{2}, \overline{\mathbf{W}_{2}}\right)\right\|_{\mathcal{Z}_{\eta}^{r}},
\end{aligned}
$$

provided $\mu$ and $\delta$ are sufficiently small and choosing $a$ as in equation (3.42).

Therefore, we have proved that for sufficiently small $\mu$ and $\delta$, we can take $a$ properly such that $\mathcal{T}_{4}$ is a uniform contraction. By fixed point theorem, for $\mu$ and $\delta$ sufficiently small, there exists a function $\mathbf{W}\left(\mu^{1 / 2}, \delta\right)$ solving equation

$$
\mathcal{T}_{4}\left(\mathbf{W}, \overline{\mathbf{W}}, \mu^{1 / 2}, \delta\right)=(\mathbf{W}, \overline{\mathbf{W}}) .
$$

Due to the smoothness of $\mathcal{T}_{4}$ away from $\mu=0$, we conclude that $\mathbf{W}\left(\mu^{1 / 2}, \delta\right)$ is smooth for $\mu \neq 0$. On the other hand, from equation (3.42), we have

$$
\lim _{\mu \longrightarrow 0+} a=0
$$

which implies that

$$
\lim _{\mu \longrightarrow 0+} \mathbf{W}\left(\mu^{1 / 2}, \delta\right)=0 .
$$

Noting that $\mathbf{W}(0, \delta)=0$, we thus proved the continuity of $\mathbf{W}\left(\mu^{1 / 2}, \delta\right)$.

The result in Theorem 1 is an immediate consequence of Theorem 2. Since $\varepsilon\left(\mu^{1 / 2}, \delta\right)$ is a continuous function with $\varepsilon(0,0)=0$, we find that the angle of the selected grain boundary is a continuous function $\alpha\left(\mu^{1 / 2}, \delta\right)$ with $\alpha(0,0)=1$, and that the wavenumber of the asymptotic rolls is $k_{x} \sim k_{x, 1}$. As a result, the wavenumber function $k\left(\mu^{1 / 2}, \delta\right)=\sqrt{k_{x}^{2}+\left(k_{*}+\delta\right)^{2}}$ with $k(0,0)=1$ is continuous, which, combined with the compactness of the interval $\left[\epsilon_{\varphi}, \pi-\epsilon_{\varphi}\right]$ for fixed $\epsilon_{\varphi} \in(0, \pi)$, shows that Theorem 1 is true.

\section{Appendix}

Lemma 4.1 There exists a positive constant $N \geqslant 1$ such that

$$
\begin{array}{r}
\left\|\partial_{x}(\widetilde{\mathcal{S}}(\mu, \delta))^{-1} f\right\|_{L_{\eta}^{2}} \leqslant \frac{N}{\mu^{1 / 2}}\|f\|_{L_{\eta}^{2}}, \\
\left\|\partial_{x}^{2}(\widetilde{\mathcal{S}}(\mu, \delta))^{-1} f\right\|_{L_{\eta}^{2}} \leqslant \frac{N}{\mu}\|f\|_{L_{\eta}^{2}}, \\
\left\|\partial_{x}^{3}(\widetilde{\mathcal{S}}(\mu, \delta))^{-1} f\right\|_{L_{\eta}^{2}} \leqslant \frac{N}{\mu^{3 / 2}}\|f\|_{L_{\eta}^{2}} .
\end{array}
$$

Proof. We define

$$
\begin{aligned}
\mathcal{J}: L_{\eta}^{2} & \longrightarrow H_{\eta}^{4} \\
V & \longmapsto \int_{\mathbb{R}} G(x-y, g(x-y)) V(y) d y
\end{aligned}
$$


where $G(x, a)=\int_{\mathbb{R}} \frac{e^{-i t x}}{1 / \mu\left(\mu t^{2}-k_{x, \ell_{*}, \delta}^{2}\right)^{2}+a} d t, \quad g(x)=6\left(C_{+}^{* 2}+C_{-}^{* 2}\right)-1$.

We note that

$$
\begin{aligned}
& G(x, a)= \begin{cases}\frac{\pi}{2} \frac{1}{\mu^{1 / 4} a^{1 / 2}\left(b^{2}+a\right)^{1 / 4}} e^{\left(\frac{\sqrt{b^{2}+a}-b}{2}\right)^{1 / 2} \mu^{-1 / 4} x}\left(\left(\frac{\sqrt{b^{2}+a}+b}{2 \sqrt{b^{2}+a}}\right)^{1 / 2} \cos \left(\left(\frac{\sqrt{b^{2}+a}+b}{2}\right)^{1 / 2} \mu^{-1 / 4} x\right)\right. & x \leqslant 0, \\
\left.-\left(\frac{\sqrt{b^{2}+a}-b}{2 \sqrt{b^{2}+a}}\right)^{1 / 2} \sin \left(\left(\frac{\sqrt{b^{2}+a}+b}{2}\right)^{1 / 2} \mu^{-1 / 4} x\right)\right), & x \geqslant 0, \\
\frac{\pi}{2} \frac{1}{\mu^{1 / 4} a^{1 / 2}\left(b^{2}+a\right)^{1 / 4}} e^{-\left(\frac{\sqrt{b^{2}+a}-b}{2}\right)^{1 / 2} \mu^{-1 / 4} x}\left(\left(\frac{\sqrt{b^{2}+a}+b}{2 \sqrt{b^{2}+a}}\right)^{1 / 2} \cos \left(\left(\frac{\sqrt{b^{2}+a}+b}{2}\right)^{1 / 2} \mu^{-1 / 4} x\right)\right. & \\
\left.+\left(\frac{\sqrt{b^{2}+a}-b}{2 \sqrt{b^{2}+a}}\right)^{1 / 2} \sin \left(\left(\frac{\sqrt{b^{2}+a}+b}{2}\right)^{1 / 2} \mu^{-1 / 4} x\right)\right), & x \geqslant 0\end{cases} \\
& G^{(1,0)}(x, a)= \begin{cases}-\frac{\pi}{2} \frac{1}{\mu^{1 / 2} a^{1 / 2}} e^{\left(\frac{\sqrt{b^{2}+a}-b}{2}\right)^{1 / 2} \mu^{-1 / 4} x} \sin \left(\left(\frac{\sqrt{b^{2}+a}+b}{2}\right)^{1 / 2} \mu^{-1 / 4} x\right), & x \leqslant 0, \\
-\frac{\pi}{2} \frac{1}{\mu^{1 / 2} a^{1 / 2}} e^{-\left(\frac{\sqrt{b^{2}+a}-b}{2}\right)^{1 / 2} \mu^{-1 / 4} x} \sin \left(\left(\frac{\sqrt{b^{2}+a}+b}{2}\right)^{1 / 2} \mu^{-1 / 4} x\right), & x \geqslant 0,\end{cases} \\
& G^{(2,0)}(x, a)= \begin{cases}-\frac{\pi}{2} \frac{\left(b^{2}+a\right)^{1 / 4}}{\mu^{3 / 4} a^{1 / 2}} e^{\left(\frac{\sqrt{b^{2}+a}-b}{2}\right)^{1 / 2} \mu^{-1 / 4} x}\left(\left(\frac{\sqrt{b^{2}+a}+b}{2 \sqrt{b^{2}+a}}\right)^{1 / 2} \cos \left(\left(\frac{\sqrt{b^{2}+a}+b}{2}\right)^{1 / 2} \mu^{-1 / 4} x\right)\right. & \\
\left.+\left(\frac{\sqrt{b^{2}+a}-b}{2 \sqrt{b^{2}+a}}\right)^{1 / 2} \sin \left(\left(\frac{\sqrt{b^{2}+a}+b}{2}\right)^{1 / 2} \mu^{-1 / 4} x\right)\right), & x \leqslant 0, \\
-\frac{\pi}{2} \frac{\left(b^{2}+a\right)^{1 / 4}}{\mu^{3 / 4} a^{1 / 2}} e^{-\left(\frac{\sqrt{b^{2}+a}-b}{2}\right)^{1 / 2} \mu^{-1 / 4} x}\left(\left(\frac{\sqrt{b^{2}+a}+b}{2 \sqrt{b^{2}+a}}\right)^{1 / 2} \cos \left(\left(\frac{\sqrt{b^{2}+a}+b}{2}\right)^{1 / 2} \mu^{-1 / 4} x\right)\right. & \\
\left.-\left(\frac{\sqrt{b^{2}+a}-b}{2 \sqrt{b^{2}+a}}\right)^{1 / 2} \sin \left(\left(\frac{\sqrt{b^{2}+a}+b}{2}\right)^{1 / 2} \mu^{-1 / 4} x\right)\right), & x \geqslant 0,\end{cases} \\
& G^{(3,0)}(x, a)= \begin{cases}-\frac{\pi}{2} \frac{\left(b^{2}+a\right)^{1 / 2}}{\mu a^{1 / 2}} e^{\left(\frac{\sqrt{b^{2}+a}-b}{2}\right)^{1 / 2} \mu^{-1 / 4} x .} & \left(\frac{\sqrt{a}}{\sqrt{b^{2}+a}} \cos \left(\left(\frac{\sqrt{b^{2}+a}+b}{2}\right)^{1 / 2} \mu^{-1 / 4} x\right)-\frac{b}{\sqrt{b^{2}+a}} \sin \left(\left(\frac{\sqrt{b^{2}+a}+b}{2}\right)^{1 / 2} \mu^{-1 / 4} x\right)\right), \quad x \leqslant 0, \\
\frac{\pi}{2} \frac{\left(b^{2}+a\right)^{1 / 2}}{\mu a^{1 / 2}} e^{-\left(\frac{\sqrt{b^{2}+a}-b}{2}\right)^{1 / 2} \mu^{-1 / 4} x} & \\
\left(\frac{\sqrt{a}}{\sqrt{b^{2}+a}} \cos \left(\left(\frac{\sqrt{b^{2}+a}+b}{2}\right)^{1 / 2} \mu^{-1 / 4} x\right)+\frac{b}{\sqrt{b^{2}+a}} \sin \left(\left(\frac{\sqrt{b^{2}+a}+b}{2}\right)^{1 / 2} \mu^{-1 / 4} x\right)\right), & x>0,\end{cases}
\end{aligned}
$$

where $b=\frac{k_{x, \ell_{*}, \delta}^{2}}{\mu^{1 / 2}}$. Then, it is not hard to see from expressions (4.2) that there exists some positive constant $C_{1} \geqslant 1$ such that

$$
\begin{array}{r}
\left\|G^{(0, j)}(x, g(x))\right\|_{L^{1}} \leqslant C_{1}, \text { for } j=0,1,2,3,4, \\
\left\|G^{(1, j)}(x, g(x))\right\|_{L^{1}} \leqslant \frac{C_{1}}{\mu^{1 / 2}}\left(\mu+k_{x, \ell_{*}, \delta}^{4}\right)^{1 / 4}, \text { for } j=0,1,2,3, \\
\left\|G^{(2, j)}(x, g(x))\right\|_{L^{1}} \leqslant \frac{C_{1}}{\mu}\left(\mu+k_{x, \ell_{*}, \delta}^{4}\right)^{1 / 2}, \text { for } j=0,1,2, \\
\left\|G^{(3, j)}(x, g(x))\right\|_{L^{1}} \leqslant \frac{C_{1}}{\mu^{3 / 2}}\left(\mu+k_{x, \ell_{*}, \delta}^{4}\right)^{3 / 4} \text {, for } j=0,1 .
\end{array}
$$

Moreover, from estimates (4.3) and by Young's inequality, for $\eta>0$ sufficiently small, there exists a positive constant $C_{2} \geqslant 1$ such that

$$
\begin{gathered}
\left\|\partial_{x} \mathcal{J}(\mu, \delta) f\right\|_{L_{\eta}^{2}} \leqslant \frac{C_{2}}{\mu^{1 / 2}}\|f\|_{L_{\eta}^{2}} \\
\left\|\partial_{x}^{2} \mathcal{J}(\mu, \delta) f\right\|_{L_{\eta}^{2}} \leqslant \frac{C_{2}}{\mu}\|f\|_{L_{\eta}^{2}} \\
\left\|\partial_{x}^{3} \mathcal{J}(\mu, \delta) f\right\|_{L_{\eta}^{2}} \leqslant \frac{C_{2}}{\mu^{3 / 2}}\|f\|_{L_{\eta}^{2}}
\end{gathered}
$$

To prove the lemma, we just need to show that for sufficiently small $\mu$ and $\delta, \widetilde{\mathcal{S}} \mathcal{J}: L_{\eta}^{2} \longrightarrow L_{\eta}^{2}$ is uniformly bounded and invertible. In fact,

$$
\widetilde{\mathcal{S}} \mathcal{J}=\mathrm{id}+\mathcal{K}_{1}+\mathcal{K}_{2}
$$


where

$$
\begin{aligned}
\left(\mathcal{K}_{1} V\right)(x)= & 2 k_{x, \ell_{*}, \delta}^{2} \int_{\mathbb{R}}\left(g^{\prime \prime}(x-y) G^{(0,1)}(x-y, g(x-y))+g^{\prime}(x-y)^{2} G^{(0,2)}(x-y, g(x-y))\right) V(y) d y+ \\
& \mu \int_{\mathbb{R}}\left(g^{(4)}(x-y) G^{(0,1)}(x-y, g(x-y))+4 g^{(3)}(x-y) G^{(1,1)}(x-y, g(x-y))+\right. \\
& 3 g^{\prime \prime}(x-y)^{2} G^{(0,2)}(x-y, g(x-y))+g^{\prime}(x-y)^{4} G^{(0,4)}(x-y, g(x-y))+ \\
& 4 g^{\prime}(x-y)^{3} G^{(1,3)}(x-y, g(x-y))+6 g^{\prime}(x-y)^{2} G^{(2,2)}(x-y, g(x-y))+ \\
& 4 g^{(3)}(x-y) g^{\prime}(x-y) G^{(0,2)}(x-y, g(x-y))+ \\
& 6 g^{\prime \prime}(x-y)\left(g^{\prime}(x-y)^{2} G^{(0,3)}(x-y, g(x-y))+2 g^{\prime}(x-y) G^{(1,2)}(x-y, g(x-y))+\right. \\
& \left.\left.G^{(2,1)}(x-y, g(x-y))\right)\right) V(y) d y . \\
\left(\mathcal{K}_{2} V\right)(x)= & \int_{\mathbb{R}}\left(4 g^{\prime}(x-y)\left(k_{x, \ell_{*}, \delta}^{2} G^{(1,1)}+\mu G^{(3,1)}\right)(x-y, g(x-y))\right) V(y) d y .
\end{aligned}
$$

By Young's inequality and estimates (4.3), it is straightforward to conclude that the norm of $\mathcal{K}_{1}$ as an operator from $L_{\eta}^{2}$ to $L_{\eta}^{2}$ goes to 0 uniformly as $\mu>0$ and $\delta$ go to zero. On the other hand, we denote $H(x, a)=k_{x, \ell_{*}, \delta}^{2} G^{(1,0)}(x, a)+\mu G^{(3,0)}(x, a)$ and have

$$
H(x, a)= \begin{cases}-\frac{\pi}{2} e^{\left(\frac{\sqrt{b^{2}+a}-b}{2}\right)^{1 / 2} \mu^{-1 / 4} x} \cos \left(\left(\frac{\sqrt{b^{2}+a}+b}{2}\right)^{1 / 2} \mu^{-1 / 4} x\right), & x \leqslant 0 \\ \frac{\pi}{2} e^{-\left(\frac{\sqrt{b^{2}+a}-b}{2}\right)^{1 / 2} \mu^{-1 / 4} x} \cos \left(\left(\frac{\sqrt{b^{2}+a}+b}{2}\right)^{1 / 2} \mu^{-1 / 4} x\right), & x>0\end{cases}
$$

Moreover, it is not hard to see that there exists some positive constant $C_{3} \geqslant 1$ such that

$$
\left\|H^{(0,1)}(x, a)\right\|_{L^{1}} \leqslant C_{3}\left(\mu+k_{x, \ell_{*}, \delta}^{4}\right)^{1 / 4}
$$

which, combined with Young's inequality, also shows that the norm of $\mathcal{K}_{2}$ as an operator from $L_{\eta}^{2}$ to $L_{\eta}^{2}$ goes to 0 uniformly as $\mu>0$ and $\delta$ go to zero.

Thus, for sufficiently small $\mu$ and $\delta$, we have

$$
\widetilde{\mathcal{S}}^{-1}=\mathcal{J}(\widetilde{\mathcal{S}} \mathcal{J})^{-1},
$$

which, combined with estimate $(4.4)$ and the fact that $(\widetilde{\mathcal{S}} \mathcal{J})^{-1}$ is uniformly bounded, shows that the lemma holds.

\section{References}

[1] V. I. Arnold, Matrices depending on parameters, Uspehi Mat. Nauk, 26 (1971), pp. 101-114.

[2] N. M. Ercolani, R. Indik, A. C. Newell, and T. Passot, The geometry of the phase diffusion equation, J. Nonlinear Sci., 10 (2000), pp. 223-274.

[3] N. M. Ercolani and S. C. Venkataramani, A variational theory for point defects in patterns, J. Nonlinear Sci., 19 (2009), pp. 267-300.

[4] M. Haragus And G. Iooss, Local bifurcations, center manifolds, and normal forms in infinitedimensional dynamical systems, Universitext, Springer-Verlag London Ltd., London, 2011. 
[5] M. Haragus And A. Scheel, Dislocations in an anisotropic Swift-Hohenberg equation, Comm. Math. Phys. to appear.

[6] _ Grain boundaries in the Swift-Hohenberg equation, Europ. J. Appl. Math. to appear.

[7] _ Interfaces between rolls in the Swift-Hohenberg equation, Int. J. Dyn. Syst. Differ. Equ., 1 (2007), pp. 89-97.

[8] B. A. Malomed, A. A. Nepomnyashchy, And M. I. Tribelsky, Domain boundaries in convection patterns, Phys. Rev. A (3), 42 (1990), pp. 7244-7263.

[9] A. Mielke, Instability and stability of rolls in the Swift-Hohenberg equation, Comm. Math. Phys., 189 (1997), pp. 829-853.

[10] B. Sandstede And A. Scheel, Relative Morse indices, Fredholm indices, and group velocities, Discrete Contin. Dyn. Syst., 20 (2008), pp. 139-158.

[11] G. J. B. van den Berg And R. C. A. M. van der Vorst, A domain-wall between single-mode and bimodal states, Differential Integral Equations, 13 (2000), pp. 369-400. 